\title{
Efficacy-enhanced and cytokine release syndrome- attenuated anti-CD7 universal chimeric antigen receptor-T cell therapy for relapsed/refractory CD7-positive hematological malignancies: A phase I clinical study
}

He Huang ( $\nabla$ huanghe@zju.edu.cn )

The First Affiliated Hospital, School of Medicine, Zhejiang University https://orcid.org/0000-0002-2723-1621

Yongxian $\mathrm{Hu}$

Bone Marrow Transplantation Center, The First Affiliated Hospital, School of Medicine, Zhejiang University

\section{Yali Zhou}

Nanjing Bioheng Biotech Co., Ltd

\section{Mingming Zhang}

Bone Marrow Transplantation Center, The First Affiliated Hospital, School of Medicine, Zhejiang University Houli Zhao

Bone Marrow Transplantation Center, The First Affiliated Hospital, School of Medicine, Zhejiang University

\section{Guoqing Wei}

Bone Marrow Transplantation Center, The First Affiliated Hospital, School of Medicine, Zhejiang University

\section{Wengang Ge}

Nanjing Bioheng Biotech Co., Ltd

\section{Gong Chen}

Nanjing Bioheng Biotech Co., Ltd

\section{Lu Han}

Nanjing Bioheng Biotech Co., Ltd

\section{Tingting Guo}

Nanjing Bioheng Biotech Co., Ltd

Jiazhen Cui

Bone Marrow Transplantation Center, The First Affiliated Hospital, School of Medicine, Zhejiang University

\section{Xiaoyan Jiang}

Nanjing Bioheng Biotech Co., Ltd

\section{Xiujun Zheng}

Nanjing Bioheng Biotech Co., Ltd

Shuhui Yu

Nanjing Bioheng Biotech Co., Ltd

\section{Xiaolong Li}

Nanjing Bioheng Biotech Co., Ltd

\section{Xingwang Zhang}

Nanjing Bioheng Biotech Co., Ltd

\section{Mingxi Chen}

Nanjing Bioheng Biotech Co., Ltd 


\section{Xiuju Li}

Nanjing Bioheng Biotech Co., Ltd

\section{Ming Gao}

Nanjing Bioheng Biotech Co., Ltd

\section{Kang Wang}

Nanjing Bioheng Biotech Co., Ltd

\section{Xiaohong $\mathrm{He}$}

Nanjing Bioheng Biotech Co., Ltd

\section{Yanbin Wang}

Nanjing Bioheng Biotech Co., Ltd

\section{Jiangtao Ren}

Nanjing Bioheng Biotech Co., Ltd

\section{Article}

Keywords: CD7, Universal CAR-T, CRISPR/Cas9, Hematological malignancy, Efficacy; Cytokine release syndrome Posted Date: May 25th, 2021

DOI: https://doi.org/10.21203/rs.3.rs-514812/v1

License: (c) (i) This work is licensed under a Creative Commons Attribution 4.0 International License. Read Full License 


\section{Abstract}

Chimeric antigen receptor-T cell (CAR-T) therapy in T cell malignancies faces fratricide, $T$ cell aplasia, and product contamination. Here, we successfully developed a universal anti-CD7 CAR-T product (RD13-01). RD13-01 contains a bbzg-CAR comprising an anti-CD7 single-chain variable fragment, a 4-1BB costimulatory domain, a CD3 3 signaling domain, the intracellular domain of the common y chain, and a natural killer cell inhibitory molecule (E-cadherin). TRAC, CD7 and HLA-II were disrupted to avoid graft versus host disease (GvHD), fratricide and rejection. Bbzg-CAR-T exerted antitumor effects superior to those of conventional CAR-T, while exhibiting reduced cytokine production. Among 11 evaluable relapsed/refractory ( $r / r)$ patients, no dose-limited toxicity, GvHD, immune effector cellassociated neurotoxicity or severe cytokine release syndrome (grade $\geq 3$ ) occurred. Nine (82\%) showed objective response. For $\mathrm{r} / \mathrm{r}$ leukemia and $\mathrm{NHL}$, complete response rates were $75 \%$ and $33.3 \%$ respectively. Outstanding safety and efficacy of this universal CAR-T product was achieved in $\mathrm{CD} 7^{+} \mathrm{T}$ cell malignancies.

\section{Introduction}

T cell malignancies are a type of highly aggressive hematological tumors generally associated with poor prognosis $^{1,2}$. While intensive chemotherapy regimens have led to improved survival, the event-free and overall survival remains lower than 70\%; relapsed or refractory ( $r / r)$ disease has particularly dismal outcomes with 5 -year overall survival (OS) rate lower than $20 \%^{3}$. Currently there are no potential curative options beyond hematopoietic cell transplantation and conventional chemotherapy, the latter of which is associated with great trade-offs in toxicities; thus, reinforcing the need for novel targeted therapies ${ }^{4}$.

Chimeric antigen receptor-T cell (CAR-T) therapies targeting CD19 have shown adequate response rates and durable remission in patients with $\mathrm{r} / \mathrm{r}$ B cell leukemia or lymphoma ${ }^{5-9}$. Cluster of differentiation (CD) 7 is a transmembrane glycoprotein expressed by $\mathrm{T}$ and natural killer (NK) cells as well as their precursors. Furthermore, CD7 is expressed in more than $95 \%$ of lymphoblastic T cell leukemias, lymphomas, and a subset of peripheral T cell lymphomas, thus making it a potential target in T cell malignancies that might allow development of promising strategies to overcome host immune rejection of allogeneic T cell therapy ${ }^{10}$. However, challenges in adapting CAR technology for $\mathrm{T}$ cell disease include fratricide, T cell aplasia, and product contamination ${ }^{11}$. Moreover, autologous CAR-T cells have certain logistic and clinical limitations ${ }^{12}$. However, an off-the-shelf allogeneic CAR-T cell product could potentially address these issues. Recently, Reuben and colleagues showed that universal anti-CD19 CAR-T cells exhibited in vivo expansion and anti-leukemic activity with a manageable safety profile in heavily pretreated patients with $r / r$ B-cell acute lymphoblastic leukemia (ALL) ${ }^{13}$. Previously, we showed that CRISPR/Cas9-engineered universal CD19/CD22 CAR-T cells exhibit a manageable safety profile and prominent anti-leukemia activity in $r / r$ B-cell ALL $^{14}$. An investigator-initiated study that used allogeneic anti-CD7 CAR (CAR7)-T cells showed deep and durable responses in two $\mathrm{r} / \mathrm{r}$ T-ALL patients, with one patient being in ongoing remission for more than one year as indicated by the data cutoff date ${ }^{15}$. But potential rejection mediated by $C D 7^{-}$host $\mathrm{T}$ and NK cells limited CAR-T cell persistence and efficacy. Enhanced lymphodepletion pre-conditioning applied to reduce host rejection and to enhance CAR-T cell efficacy increased the incidence of infection. High frequency of severe CRS occurred during this study also posed another concern for clinical application.

Here, we aimed to develop a universal anti-CD7 CAR-T cell product (RD13-01) which is an off-the-shelf, efficacyenhanced, CRS-attenuated, rejection-resistant CAR-T cell product. We produced RD13-01 by transducing allogeneic $T$ cells via a retroviral vector encoding a third-generation CAR incorporating an anti-CD7 single-chain variable fragment (scFv), a 4-1BB costimulatory motif, a CD3ろ signaling domain, and an intracellular domain of the cytokine common 
Y chain (Yc) (bbzg-CAR). CRISPR/Cas9 was used to knock out the genes encoding the T cell receptor (TCR), human leucocyte antigen (HLA) class II, and CD7 to minimize the risks of graft versus host disease (GvHD), $C D 7^{-} \mathrm{CD} 4^{+} \mathrm{T}$ cell rejection, and fratricide among CAR-T cells. In addition, an NK cell inhibitor (NKi) structure was introduced to inhibit CD7 NK cells, to further prevent rejection. Preclinical data demonstrated the safety and efficacy of RD13-01. We also conducted a phase I clinical trial using RD13-01 in patients with r/r CD7 malignancies (NCT04538599).

\section{Results}

\section{CD3, HLA-II, and CD7 knockout (KO) and NK cell inhibitor overexpression to construct rejection-resistant "off-the- shelf" anti-CD7 CAR-T cells}

We discovered that TCR/CD3 and CD7 expression could be efficiently eliminated using the CRISPR/Cas9 technology to generate GvHD deficient CAR7 T cells (Fig. 1a). These cells (bbz-37KO) demonstrated robust tumor antigenspecific killing and secreted cytokines that promoted CAR-T cell proliferation as well as effector function (Extended Data Fig. 1a, b), and exhibited better tumor growth control and significantly prolonged mouse survival than nontransduced $\mathrm{T}$ cells (Fig. $1 \mathrm{~b}, \mathrm{c}$ ). All the $\mathrm{CD} 8^{+} \mathrm{T}$ cells were $\mathrm{CD} 7^{+}$and, although a minority $(\sim 5 \%)$ subset of $\mathrm{CD} 4^{+} \mathrm{T}$ cells were $\mathrm{CD}^{-}$in healthy individuals (Fig. 1d), this population was more prominent ( 20\%) in the CD $4^{+}$subset in T-ALL patients. And these $\mathrm{CD} 7^{-} \mathrm{CD} 4^{+} \mathrm{T}$ cells can cause alloreactive rejection. Thus, we ablated HLA-II by disrupting RFX5 to diminish CD4 ${ }^{+} \mathrm{T}$ cell alloreactivity (Fig. 1e). Another aspect impacting allogeneic CAR-T cell persistence is CD7- NK cell surveillance through missing-self recognition. E-cadherin is an NKi that functions via KLRG1 binding ${ }^{16}$. Therefore, we engineered CAR7 T cells with the EC1-EC2 extracellular domain of E-cadherin fused to a CD28 costimulatory domain, which might inhibit NK cell lysis and switch the adhesion signal to pro-survival signal. NKimodified cells showed enhanced resistance to NK cell rejection (Fig. 1f).

\section{Addition of Yc improves CAR-T cell anti-leukemia activity by activating JAK/STAT3}

Interleukin (IL)-2 is a pivotal cytokine required by CAR-T cells for their robust expansion and effector function ${ }^{17,18}$. However, the absolute IL-2 secretion level of bbz-37KO CAR7 T cells following tumor challenge was below that of the well-examined CAR19 T cell models, and the IL-2 production in CD7 KO CAR19 T cells was already decreased to 1/4 of its wild-type counterparts (Extended Data Fig. 2a). This is consistent with the finding that CD7 expression is critical for IL-2 production ${ }^{13,14}$. IL-2 secretion was not observed in CAR-T cells targeting another T cell activationassociated antigen, CD5, suggesting a shared feature of T cell antigen-directed CAR (Extended Data Fig. 2b).

Yc is a shared receptor subunit of $y c$ cytokines $^{15,16}$. Therefore, incorporating $y c$ to a CAR might bypass the IL-2 requirement. To test this hypothesis, we tethered the yc intracellular domain to conventional second-generation bbz CAR7 (Fig. 2a). Surprisingly, in the 327KO context, the addition of yc augmented IL-2 production by 3-fold and significantly enhanced cytotoxicity, suggested by exponentially elevated perforin and granzyme $A$ formation, thus indicating a positive feedback between the yc and CD7-independent IL-2 production (Fig. 2b, e). The design of rejection-resistant CAR7 bbzg-NKi T cells is illustrated in Fig. 2c. Additionally, bbzg-CAR-T cells showed higher magnitude of proliferation, as indicated by increased $\mathrm{Ki} 67^{+}$propagating-cell count, decreased apoptosis, and 1.5 -fold cell expansion compared with those of conventional CAR-T cells following tumor challenge (Fig. 2d, S2c, d). Similar findings were observed in the 37KO setting (Extended Data Fig. 2e-h). Furthermore, the percentage of STAT3 ${ }^{+}$cells was greatly enhanced in bbzg-CAR-T cells upon antigen stimulation, whereas that of STAT5 was not altered, indicating that a selective JAK-STAT downstream signaling pathway of $y c$ accounts for these enhanced properties (Fig. 2f, S2i). Bbzg-CAR-T cells express significantly lower levels of exhaustion markers, which is suggestive of the 
fitter state of bbzg-CAR-T cells (Fig. $2 \mathrm{~g}$ ). When tested in vivo, bbzg-CAR-T cells were more capable of tumor control than conventional CAR-T cells in both the $37 \mathrm{KO}$ and $327 \mathrm{KO}$ contexts (Fig. 2h, S2j-I). In addition, $\mathrm{Yc}$ also enhanced the antitumor activity of CAR19 T cells, where CD7 was intact (Extended Data Fig. 2m).

\section{Addition of yc simultaneously attenuates CRS-related cytokine production by suppressing the PI3K/AKT pathway}

Secretion of the CRS-related cytokine-interferon (IFN)- $y$, by the bbzg-CAR-T cells, was decreased to half of the bbzCAR-T cells. Furthermore, tumor necrosis factor (TNF)-a and granulocyte-macrophage colony-stimulating factor (GMCSF) levels also appeared to be reduced; expression of granzyme $B$, another granule recently demonstrated to trigger $\mathrm{CRS}^{17}$, was greatly decreased in both $\mathrm{CD} 4^{+}$and $\mathrm{CD} 8^{+} \mathrm{T}$ cells (Fig. 3a).

To gain detailed insights into the mechanisms underlying these observations, we performed RNA sequencing analysis of bbz- and bbzg-CAR7 T cells following antigen encountering. As expected, compared with bbz-CAR-T cells, mRNA levels of the JAK-STAT signaling molecules, IL-2, and yc were increased in bbzg-CAR-T cells. The mRNA levels of molecules related to T cell function (CD8b, NLRP6, TSHR, XCL1, and XCL2) as well as T cell memory (BCL6b, MVB12B, CD248, and EOMES) was also greatly elevated (Fig. 3b). While, levels of molecules associated with $T$ cell exhaustion and dysfunction, such as PDCD1, LILRB1, and LILRB4, were decreased. Furthermore, the expression of a recently identified master regulator of T cell exhaustion-TOX, was also reduced.

Interestingly, levels of molecules downstream of the PI3K/AKT pathway were greatly diminished in bbzg-CART compared with bbz-CAR-T cells, including PDGFRA, VWF, GNG7, SYK, IL6, as well as several integrins and collagens. DisGeNET analysis revealed that the PI3K/AKT pathway was perturbed by yc signaling (Fig. 3b), which may have arisen from the competition between these two pathways.

To verify these findings, we measured phosphorylation of signal transduction mediators downstream of the PI3K pathway following antigen stimulation. AKT phosphorylation was greatly reduced in bbzg-CAR7 T cells compared with that in bbz-CAR7 T cells. We found that MEK and ERK phosphorylation was reduced in bbzg-CAR-T cells, resulting in decreased phosphorylation and faster dephosphorylation of CD3ろ (Fig. 3c). Bbzg-CAR-T cells comprised more stem cell memory T cells than bbz-CAR counterparts, which is consistent with the PI3K/AKT pathway inhibition (Fig. 3d). The ability of bbzg-CAR-T cells to trigger CRS was also evaluated in human immune system reconstituted

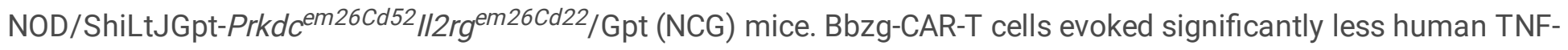
$a$, IFN- $y$, and IL- 6 secretion than bbz-CAR-T cells. Furthermore, human cytokines might activate murine immune cells ${ }^{19}$; however, levels of murine TNF-a and CCL2 production were profoundly reduced in bbzg-CAR-T cells treated group (Fig. 3e).

\section{Patient characteristics}

From September 2020 to January 2021, 12 patients with r/r CD7 ${ }^{+}$hematological malignancies, including T cell acute lymphoblastic leukemia (T-ALL), acute myeloid leukemia (AML), T cell lymphoblastic lymphoma (T-LBL), EBV ${ }^{+} \mathrm{T} / \mathrm{NK}$ cell lymphoma, peripheral T cell lymphoma, and malignancies not otherwise specified (PTCL and NOS), were enrolled in this study (Table 1). CAR7bbzg-NKi (RD13-01) T cells were manufactured from allogeneic third-party healthy donor peripheral blood mononuclear cells in advance, and no karyotypic abnormalities were observed in them (Extended Data Fig. 3a). No CD7:TRAC, TRAC:CD7, RFX5:TRAC, or RFX5:CD7 chromosomal rearrangements were detected. CD7:RFX5 and TRAC:RFX5 rearrangements were present in less than $0.02 \%$ of the harvested cells (Extended Data Fig. 3b). The 12 patients received lymphodepletion chemotherapy (Fludarabine $30 \mathrm{mg} / \mathrm{m}^{2}$ on day -7

to -3 , cyclophosphamide $300 \mathrm{mg} / \mathrm{m}^{2}$ on day -7 to -3 , and etoposide $100 \mathrm{mg} /$ day on day -7 to -3 ) followed by RD13-01 
infusion (day 0): three patients received $1 \times 10^{7}$ cells $/ \mathrm{kg}$, six patients received $2 \times 10^{7} \mathrm{cells} / \mathrm{kg}$, and the other three received $3 \times 10^{7}$ cells $/ \mathrm{kg}$. The detailed information of RD13-01 is provided in Extended Data Table 1. The median patient age was 34 (range between 8 and 66), and the median prior lines of therapies were four (range between 2 and 5). The 12 patients were previously treated with at least two lines of chemotherapy while three of them had undergone hematopoietic stem cell transplantation (HSCT) previously, including two allogeneic HSCT and one autologous HSCT (Extended Data Table 2). Four patients reported previous central nervous system (CNS) involvement. Of the eight patients with leukemia, four exhibited extramedullary disease while the other four patients with lymphoma exhibited bone marrow involvement. Of the 10 patients with bone marrow involvement, the median bone marrow blast was 29\% (range between $7 \%$ and $95 \%$ ).

\section{RD13-01 safety profile}

No dose-limited toxicity, GvHD, or immune effector cell-associated neurotoxicity (ICANS) have occurred in the patients to March $31^{\text {st }} 2021$ (Fig. 4c). Adverse events in this study are summarized in Extended Data Table 3. CRS of any grade occurred in 10 of 12 patients but none experienced severe CRS (grade $\geq 3$ ) (Fig. 4c). The median time of CRS onset was 1 day. Levels of cytokines associated with CRS, including IL-6, IL-10, and IFN-ץ, increased significantly in patients who developed CRS (Fig. 4d). For CRS management, 4 and 1 patients received tocilizumab and corticosteroid, respectively, whereas three patients received a combination of tocilizumab and corticosteroid. Patient 3 developed sepsis and died 24 days after the infusion.

EBV-associated B-cell lymphoproliferation (EBV-LPD) was identified in one patient (patient 6). This 34-year-old male patient with PTCL-NOS achieved complete remission (CR) on day 28 following RD13-01 infusion and was diagnosed with EBV-associated DLBCL on day 55. The patient received rituximab-based therapeutic regimen and finally died of progressive disease. Monitoring of EBV-DNA after CAR-T infusion was performed in 5 of 12 patients with baseline EBV viremia. In these five patients, the load of EBV-DNA declined during CAR-T expansion, and EBV reactivation was subsequently confirmed following elevation of EBV-DNA copy number to baseline levels (Extended Data Fig. 4).

\section{RD13-01 efficacy}

Eleven of the 12 patients survived for 28 days or more, of whom nine (82\%) achieved a response (Fig. 4e). Of the eight patients with leukemia, six (75\%) achieved CR/CR with incomplete hematological recovery (CRi) and showed minimal residual disease-negative CR (MRD-CR) (Fig. 4f). With a median follow up of 100 days in patients with leukemia, the median leukemia-free survival was estimated at 72 days. Patients 1, 4, and 5 experienced relapses on day 100,103, and 64 after the CAR-T cell infusion, respectively. Patient 7 failed to achieve a response and died on day 64 after the infusion owing to disease progression and subsequent infection. The three patients with lymphoma

achieved a response after infusion, among whom patient 6 achieved complete response whereas patients 10 and 12 achieved partial response (Figure 4f). However, patient 6 developed EBV-associated DLBCL after achieving CR to RD13-01 and died 93 days after the infusion.

\section{RD13-01 cellular pharmacokinetics}

Using quantitative polymerase chain reaction (qPCR), RD13-01 expansion was observed in all patients and peaked between day 7 and 28 in responders (Fig. 5a). The mean peak expansion was 1,729,247 copies/ $\mu$ g genomic DNA in responders whereas it was 9,054 and 1,330,908 copies/ $\mu$ g genomic DNA in two nonresponding patients. Using FCM too, expansion was observed in all patients. The nine patients who responded by day 28 exhibited a peaked absolute $\mathrm{CAR}^{+}$RD13-01 cells in their peripheral blood between days 7 and 14 (Fig. 5b). The median peaked absolute CAR+ 
RD13-01 cells count was 143.49 (range, 21.66 to 742.63) cells/ $\mu \mathrm{L}$ in responders whereas it was 54.53 and 100.85 cells/ $\mu \mathrm{L}$ in two nonresponding patients, respectively. The median duration of RD13-01 persistence measured using qPCR was 28 days after infusion (range between 10 and 120 days). Of the two non-responders, low in vivo expansion of RD13-01 led to lack of response in patient 2, whereas RD13-01 expansion was observed in the peripheral blood of patient 7 with extramedullary disease, indicating that the tumor microenvironment exerted an immunosuppressive effect.

Following RD13-01 infusion, all 12 patients showed grade 3/4 neutrophil-count decrease, and the nine responders developed CD7+ T lymphocytopenia; intrinsic CD7- T cell count increased at a median of 63.93 cells/ $\mu \mathrm{L}$ (range, 21.92 to 609.46$)$ by day 28 (Fig. 5c-f).

\section{Discussion}

Universal CAR-T cells modified using gene-editing techniques have attracted considerable attention owing to their high quality, prompt availability, and widespread application for patients. RD13-01 is an allogeneic, healthy donorderived, and CRISPR/Cas9-edited anti-CD7 CAR-T cell product. It could prevent fratricide and host rejection owing to its unique structure characterized by TRAC/CD7/HLA-II KO and NKi overexpression. The efficacy enhanced and rejection-resistant product eliminated the need of intensive lymphodepletion used in the previous study, thus deceased the risk of infection. RD13-01 was also able to attenuate CRS-related cytokine production and enhance anti-leukemia activity in vitro due to yc presence. Moreover, this study, which is the first-in-human, single-arm, doseescalation study showed that RD13-01 exhibits a notable safety profile and achieved high response rate in patients with $\mathrm{r} / \mathrm{r}$ T cell malignancies.

In our study, rapid tumor elimination and deep remission were observed for all dose levels and CD $7^{+}$disease types, including leukemia and lymphoma. Furthermore, RD13-01 showed prominent antitumor activity in patients with extramedullary relapse. This clinical data further demonstrated the rejection resistance and enhanced efficacy of "off-the-shelf" anti-CD7 CAR-T cells. Interestingly, one patient with CD7 ${ }^{+} \mathrm{r} / \mathrm{r}$ AML received compassionate therapy with RD13-01 and achieved MRD ${ }^{-}$CR. While CD7 is expressed in approximately $30 \%$ of patients with AML and is associated with aggressive disease phenotypes and overall poor prognosis ${ }^{20-22}$, the potential clinical feasibility of anti-CD7 CAR-T therapy in AML was previously shown in a preclinical study ${ }^{23}$. Our study provided further evidence for the potential feasibility of anti-CD7 CAR-T therapy against AML. Nevertheless, additional studies for further validation are necessary.

Unlike CD19-targeted CAR-T therapy, CD7 have to be abrogated to prevent T cell fratricide for CD7-directed CAR-T cells. Although preclinical and clinical studies have demonstrated the potent efficacy of CAR7 T cells, we found that CD7 ablation greatly diminished IL-2 production, independent of the scFv chosen for the CAR construction (data not shown). Similar observations were made for CD7-disrupted CAR19 and CD5-disrupted CAR5 T cells, indicating an intrinsic character of T cell lineage antigen-deficient CAR-T cells. Since yc is downstream of the IL-2 signaling pathway, we speculated that by tethering yc to CAR we might bypass the IL-2 requirement. Unexpectedly, we discovered that incorporating $Y c$ into the CAR not only boosted CAR-T cell effector functions but also rescued IL-2 production. As CD7 is crucial for IL-2 production, an alternative IL-2 production pathway downstream of $\gamma \mathrm{c}$ and independent of CD7 might exist. Upregulation of STAT3 signaling is associated with complete response in patients receiving CAR19 T cell therapy ${ }^{24}$. Another intriguing finding is the downregulation of the PI3K/AKT pathway. As $\mathrm{PI} 3 \mathrm{~K} / \mathrm{AKT}$ promotes glycolysis and T cell differentiation, inhibition of PI3K/AKT during CAR-T cell expansion generates more memory like $T$ cells, thus favoring improved fitness and functions ${ }^{18,19}$. Furthermore, the Yc- $^{-}$ 
engineered CAR-T cells showed a younger phenotype and expressed less exhaustion-associated markers than conventional CAR-T cells, predictive of enforced persistence and potency. The PI3K/AKT pathway regulates MEK/ERK signaling via Rac-GEF and $\mathrm{pRex} 1^{25}$. MEK inhibition is reported to reprogram $\mathrm{CD} 8^{+} \mathrm{T}$ lymphocytes into memory stem cells with potent antitumor effects ${ }^{26}$. Interestingly, we discovered that our yc CAR-T cells also exhibit fast dephosphorylation of the MEK downstream factor ERK. Additionally, yc enhanced the function of secondgeneration CAR19 T cells. Nevertheless, whether yc exerts the same effects on other CARs remains to be investigated.

Another major challenge for CAR-T cell survival in T-ALL patients is represented by the minor population of CD4 ${ }^{+} \mathrm{CD} 7^{-}$ T-cells, which was increased to $20 \%$ in the T-ALL patients enrolled in our study. HLA-II KO is required to evade $\mathrm{CD}^{+} / \mathrm{CD} 7^{-} \mathrm{T}$ cell rejection via down-regulation of regulatory factors. A previous study showed that insertion of a nonclassical HLA molecule, HLA-E, in donor cells is sufficient to block the alloreactivity of $90 \%$ NK cells ${ }^{27}$. Nevertheless, the expression level of its receptor, NKG2A, on NK cells varies substantially among individuals ${ }^{28}$. Another drawback of this approach is represented by the possibility of HLA-E to activate NK cells through NKG2C, which is highly expressed in some hosts ${ }^{29}$. E-cadherin specifically binds to the NK cell inhibitory receptor KLRG1, which might provide a better solution for NK cell inhibition ${ }^{30}$. A previous study used an elegant design to show that a 28EZ CAR attenuated CAR-T cell activation-induced cell death (AICD) by recapitulating natural CD3Z dephosphorylation by tethering the $\mathrm{CD} 3 \varepsilon$ domain, leading to rapid $\mathrm{CD} 3 \zeta$ dephosphorylation and reduced cytokine production ${ }^{31}$. This is in line with our findings. This might contribute to the enhanced anti-leukemia effects and reduced cytokine production of the yc CAR-T cells.

CRS-related toxicity might challenge the application of CAR-T-cell therapy in the clinic. Lisi and colleagues described the course of treatment of two r/r T-ALL patients who received CD7-targeted universal CAR-T cells. Both patients exhibited grade 3 CRS complications and received ruxolitinib-based CRS management treatment ${ }^{15}$. In our study, grade 3 or higher CRS was not observed. Furthermore, the low incidence of severe CRS might be explained by the addition of $y c$ as demonstrated in vitro and in animal models.

Monocytes and macrophages are the major mediators of CRS, and IFN- $\gamma$, GM-CSF, TNF- $\mathrm{a}$, and IL- 6 are among the core activators of monocytes and macrophages ${ }^{32}$. In the setting of T cell-engaging therapies, CRS is triggered by the massive release of IFN- $\gamma$ from activated T cells or from tumor cells. The IFN- $\gamma$-activated macrophages further produce cytokines such as IL-6, TNF-a, and IL-10. GM-CSF is also a potent agonist of macrophages ${ }^{33}$. And, the GMCSF gene has also been knocked out in CAR-T cells to alleviate CRS occurrence in preclinical models ${ }^{34}$. In our study, we discovered that CRS reduction and CAR-T cell functional enhancement can be achieved by simply attaching yc to a CAR. Besides playing an important role in the induction of CRS, IL-6 potently inhibited activation and cytokine production of $\mathrm{CD}^{+} \mathrm{T}$ cells in vitro ${ }^{35}$. Thus, it may provide another potential benefit to the potency of CAR-T therapy by alleviating CRS.

Gasdermin E-mediated target cell pyroptosis induced by CAR-T cells was recently discovered to trigger CRS ${ }^{36}$. CAR-T cells rapidly activate caspase 3 in target cells by releasing granzyme B. The latter cleaves gasdermin E, a poreforming protein highly expressed in B leukemia cells, which results in extensive pyroptosis. Consequently, pyroptosisinduced factors activate caspase 1, which leads to GSDMD cleavage in macrophages and ultimately results in the release of cytokines and subsequent CRS. This might explain the lower number of CRS events in our study, as decreased granzyme $B$ release was also observed in the bbzg-CAR-T cells. 
Additionally, in effector CD8 ${ }^{+} \mathrm{T}$ cells, IL-6 is a master regulator of IL-21. Quite different from the results of a previous finding that incorporating a truncated IL-2 receptor $\beta$ chain to a CAR induced IL-21 signaling ${ }^{37}$, incorporating $\gamma c$ in our study reduced IL-21 level as well as its downstream targets, IL-18R1, IL18RBP, and IL18RAP. The response timing of activated $\mathrm{CD}^{+} \mathrm{T}$ cells to the $\mathrm{yc}$ cytokines is critical considering the study by Hinrichs and colleagues who showed that when exogenous IL-21 was added to the culture media during antigen priming, acquisition of effector functions and induction of granzyme B were inhibited, whereas exogenous IL-2 and IL-15 had an opposite effect. This may explain the discrepancy in granzyme $A$ and $B$ regulation ${ }^{38-40}$.

EBV-LPD may occur among patients with primary or secondary immunodeficiency disorders ${ }^{41}$. Furthermore, EBVLPD is a frequent event that increases the morbidity and mortality of patients undergoing HSCT or solid organ transplantation (SOT). The incidence of EBV-LPD is 1.2-12.9\% and 1-16\% after HSCT and SOT, respectively 42,43 . Additionally, EBV-LPD secondary to hematological malignancy therapy is not an uncommon event ${ }^{44-46}$. In a retrospective study that examined more than 600 patients with T cell lymphoma, 17 patients exhibited concomitant EBV-LPD at diagnosis or developed secondary EBV-LPD ${ }^{44}$. Four patients developed secondary EBV-associated DLBCL or plasmacytoma during treatment. O'Mahony and colleagues showed that for patients with T cell malignancies, 4 of 29 (13.7\%) patients developed EBV-LPD following siplizumab (an anti-CD2 antibody with high efficiency in depleting both T and NK cells) treatment. In our study, B, T, and NK cell aplasia was observed in the majority of patients due to pre-conditioning and RD13-01 therapy. It is worth mentioning that CD7-targeted CAR-T therapy might increase the incidence of EBV-LPD due to the depletion of T and NK cells in patients with T cell malignancies. Interestingly, we discovered that normal $\mathrm{CD}^{-} \mathrm{T}$ cells expanded dramatically after normal $\mathrm{CD} 7^{+} \mathrm{T}$ cells were eliminated by CD7 CAR-T cells. Thus, intrinsic CD7 $\mathrm{T}$ cells might play a compensatory role for $\mathrm{CD} 7^{+} \mathrm{T}$ lymphocytopenia, resulting in the low incidence of infection. Nevertheless, further investigation of the risk of EBVPLD during anti-CD7 CAR-T therapy is necessary.

Taken together, our preliminary data showed that RD13-01 exhibits a notable safety profile and achieved high response rates in patients with $\mathrm{r} / \mathrm{r} \mathrm{T}$ cell malignancies. This ready-for-use allogeneic CAR-T product decreased the risk of malignant T cell contamination and manufacture failure, which autologous CAR-T therapy is currently challenged by, and might provide an alternative treatment strategy for patients with $\mathrm{CD} 7^{+} \mathrm{T}$ cell malignancies. The durability of the response could not be evaluated owing to the short follow-up duration and small sample size. Further investigation should be initiated to thoroughly evaluate the clinical feasibility of CD7-targeted allogeneic CAR-T therapy.

\section{Online Methods}

\section{Construct design and RD13-01 generation}

RD13-01 contains a CAR comprising a CD7-binding scFv, a 4-1BB costimulatory domain, a CD3ろ signaling domain, and a yc intracellular domain, in addition to a EC1-EC2 extracellular domain of E-cadherin fused to a CD28 costimulatory domain. The CAR and the NKi ligand are linked by a F2A self-cleaving peptide.

The retrovirus was produced in $293 \mathrm{GP}$ cells, cryopreserved at $-80^{\circ} \mathrm{C}$, and thawed immediately before transduction. $\mathrm{CD}^{+} \mathrm{T}$ cells were isolated and activated using anti-CD3/CD28 Dynabeads (Gibco, Cat no: 40203D) following the manufacturer's protocols. The cells were cultured in X-VIVO 15 medium (Lonza, Cat no: 04-418Q) supplemented with $5 \%$ fetal bovine serum (FBS) and $300 \mathrm{IU} / \mathrm{mL}$ IL-2. Two days after T cell stimulation, the Dynabeads were removed, and CD7, TRAC, and RFX5 genes were knocked out using CRISPR/Cas9 before transduction. In brief, the T cells were 
washed twice with OPTI-MEM (Gibco, Cat no: 11058021) and resuspended in OPTI-MEM at a final concentration of $1 \sim 3 \times 10^{8}$ cells $/ \mathrm{mL}$. Subsequently, $0.4 \mathrm{~mL}$ of these cells were mixed with $120 \mu \mathrm{g}$ of Cas $9 \mathrm{mRNA}$ and electroporated into the cells using a BTX Agile Pulse apparatus. Following electroporation, the cells were immediately cultured at 37 ${ }^{\circ} \mathrm{C}$ and $5 \% \mathrm{CO}_{2}$. After $16-18 \mathrm{~h}, 40 \mu \mathrm{g}$ of total single guide (sg)RNA targeting TRAC, CD7, and RFX5 at an equal molecular ratio (Genescript) was electroporated as described below. Lastly, the T cells were centrifuged at 2,000g for $2 \mathrm{~h}$ at $32^{\circ} \mathrm{C}$ twice with the retrovirus on days 4 and 5 in retronectin-coated plates. The CAR-T cells were expanded for an extra 6 days.

The genomic sgRNA target sequences (with the protospacer adjacent motif in bold) were as follows: TRAC. 5'AGAGTCTCTCAGCTGGTACACGG-3';

CD7: 5'-GTAGACATTGACCTCCGTGATGG-3';

RFX5: 5'-GGGGTTGCGGATCCACCTATAGG-3'.

\section{In vitro cytotoxicity assay}

Jurkat-Luci tumor cells were generated and used in a luciferase-based cytotoxic T lymphocyte (CTL) assay. Briefly, a firefly luciferase-encoding lentivirus was transduced into Jurkat tumor cells to generate a parental cell for the target cell line preparation. The resulting target cells were resuspended at $1 \times 10^{5}$ cells $/ \mathrm{mL}$ in X-VIVO 15 medium and incubated with different ratios of T cells (e.g., 10:1 and 5:1) for $8 \mathrm{~h}$ at $37^{\circ} \mathrm{C}$. Subsequently, $100 \mu \mathrm{L}$ of the mixture was transferred to a 96-well white luminometer plate. Next, $100 \mu \mathrm{L}$ of substrate (Vazyme, Cat\#DD1201-01) was added, and the luminescence was immediately determined by Infinite 200 PRO (TECAN).. The results are reported as the percentage of killing based on the luciferase activity in the wells with tumor cells but no T cells [\% killing = $100-$ ((relative light units (RLU) from well with effector and target cell coculture)/(RLU from well with target cells) $\times$ 100)]. ${ }^{17}$

\section{Cytokine production assay}

A total of $1 \times 10^{5}$ CAR7 T cells/well were stimulated with Jurkat cells at a 1:1 ratio in a bottom 96-well tissue culture plate for $8 \mathrm{~h}$. Cytokines produced by CAR7 T cells were measured using an enzyme-linked immunosorbent assay (ELISA) kit and antibodies against human IL-2 (R\&D, Cat\#DY202), human IFN-Y (R\&D, Cat\#DY285B), human TNF-a (R\&D, Cat\#DY210), and human GM-CSF (R\&D, Cat\#DY215).

A total of $1 \times 10^{5}$ CD7 KO CAR19 T cells/well were stimulated with Nalm6 cells at a 1:1 ratio in a bottom 96-well tissue culture plate for 16-18 h. Cytokines produced by CD7 KO CAR19 T cells were measured using an enzymelinked immunosorbent assay (ELISA) kit and antibodies against human IL-2 and human IFN-ץ.

\section{Flow cytometry}

Anti-human CD3 FITC (Cat\#555916, DB pharmingen ${ }^{\text {TM}}$ ), CD7 PE (Cat\#343106, Biolegend), HLA-II APC (Cat\#361714, Biolegend), PD1 BV421 (Cat\#329920, Biolegend), TIM3 FITC (Cat\#345022, Biolegend), LAG3 PE (Cat\#369306, Biolegend), CD45RO APC(Cat\#304210, Biolegend), CD4 FITC (Cat\#555346, DB pharmingen ${ }^{\text {TM}}$ ), and CD8 APC (Cat\#555369, DB pharmingen ${ }^{\mathrm{TM}}$ ) antibodies were used to stain cells in phosphate-buffered saline medium. All antibodies were used according to the manufacturer's instructions. Flow cytometry was performed on a BD Celesta instrument, and the data were analyzed using the FlowJo 10 software (FlowJo, LLC). 


\section{Animal model}

5- to 7-week-old NCG mice were engrafted with Jurkat-GFP-Luci cells through intravenously (i.v.) injection, and $1 \times$ $10^{7}$ of CAR7 T cells were i.v. injected 4 days later. To measure luminescence, we injected the mice with $150 \mathrm{mg} / \mathrm{kg}$ of D-luciferin intraperitoneally and monitored tumor burden by recording luminesce in an in vivo imaging system (Caliper Life Sciences) at the indicated time points. Live imaging software was used to visualize and calculate total luminescence. To analyze the tumor cells from the peripheral blood of the mice, $100 \mu \mathrm{L}$ of blood sample was collected by orbital puncture. After red blood cell lysis, the cells were incubated for subsequent flow cytometric analysis. Survival curves were generated. Mice were euthanized when they developed signs of excessive tumor burden or when the weight loss exceeded $20 \%$ of baseline. All animal experiments were conducted in compliance with laboratory animal welfare and ethics committee.

\section{In vivo cytokine release detection}

Female, 5- to 7-week-old NCG mice received i.v. $2 \times 10^{6}$ Jurkat-GFP-Luci cells on day 0, while 6 days later, they were i.v. administered $1 \times 10^{6}$ freshly isolated peripheral blood mononuclear cells and $5 \times 10^{6} \mathrm{CAR} 7 \mathrm{~T}$ cells. To track cytokine production of both infused populations in vivo, $150 \mu \mathrm{L}$ peripheral blood was obtained by tail-vein bleeding at specified time points. Serum samples were measured using ELISA kits for human IFN-Y (R\&D, Cat\#DY285B), human G-CSF (R\&D, Cat\#DY214), human M-CSF (R\&D, Cat\#DY216), human GM-CSF (R\&D, Cat\#DY215), human TNF-a (R\&D, Cat\#DY210), human IL-1 $\beta$ (R\&D, Cat\#DY201-05), human IL-6 (R\&D, Cat\#DY206-05), mouse IL-1 $(R \& D$, Cat\#DY401), mouse IL-6 (R\&D, Cat\#DY406), mouse CCL2 (R\&D, Cat\#DY479), mouse G-CSF (R\&D, Cat\#DY414), and mouse TNF-a (R\&D, Cat\#DY410).

\section{Recipient CD4 T cell alloreactivity assay}

Donor T cells were isolated and activated using anti-CD3/CD28 Dynabeads (Gibco). These cells were further used as stimulators after HLA-II upregulation and subsequent mitomycin-c treatment. To generate primed alloreactive recipient $T$ cells, pretreated donor T cells were mixed with fresh allogeneic donor peripheral blood mononuclear cells at a 1:2 ratio in X-VIVO 15 medium supplemented with 10\% FBS and $300 \mathrm{IU} / \mathrm{mL} \mathrm{IL}-2$. IL-2 were withdrawn from day 4. Ten days later, the cells were harvested, labeled with CSFE, and cocultured with CAR7 T cells from the same donor with (327KO) or without HLA-II KO (37KO), respectively, for $6 \mathrm{~h}$. Following coculture, the cells were incubated with CD3 and CD4 antibodies and permeabilized for 10 min using BD FACS Permeabilizing Solution 2, followed by incubation with anti-IFN-y antibody and analysis using flow cytometry.

\section{NK cell alloreactivity measurement}

K562-luciferase cells were infected with a lentivirus encoding a chimeric receptor comprising an EC1-EC2 extracellular domain, an E-cadherin transmembrane domain, and the CD28 intracellular domain (K562-Ecad). Three days after infection, the engineered cells were subjected to coculture with NK92 cells. KLRG1 expression was introduced in NK92 cells through KLRG1 mRNA electroporation. Target cells were cocultured with NK92 cells at different effector:target ratios at $37^{\circ} \mathrm{C}$. Luminescence was determined immediately after $4 \mathrm{~h}$ of coculture to evaluate the NK cell cytotoxicity. To test the cytokine release from NK cells, $1 \times 10^{5} \mathrm{NK} 92$ cells/well were cocultured with K562-Ecad cells at a 1:1 ratio in a bottom 96-well tissue culture plate for $12 \mathrm{~h}$. Cytokines produced by NK92 cells were measured using a human IFN-y ELISA kit.

\section{Protein phosphorylation assay}


A total of $1 \times 10^{6}$ CAR7 T cells/well were stimulated with Jurkat-GFP cells at a 1:1 ratio in a bottom 96-well tissue culture plate. Next, $500 \mu \mathrm{L}$ of $4 \%$ paraformaldehyde in PBS was added to the cells to immediately stop the reaction at the indicated time points and the cells kept at $37^{\circ} \mathrm{C}$ for an additional $10 \mathrm{~min}$. CAR7 T cells cocultured with nontargeted K562-GFP cells were used as a baseline control. The cells were cooled on ice for 1 min and centrifuged at $400 \times \mathrm{g}$ for $5 \mathrm{~min}$ at $4{ }^{\circ} \mathrm{C}$. They were then permeabilized in $1 \mathrm{~mL}$ of ice-cold $100 \%$ methanol and incubated on ice for 30 min before washing with FACS buffer (1\% FBS in PBS) three times. Subsequently, the cells were incubated with CD3 antibody. Phospho-MEK, p-ERK, p-STAT3, and p-AKT levels were evaluated using the respective phospho-

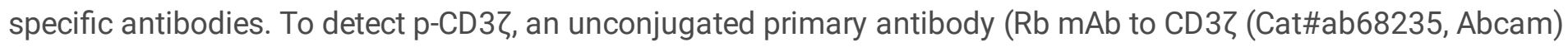
against its phospho-epitopes was incubated with the cells first, which were then further probed with a fluorescenceconjugated secondary antibody (donkey anti-rabbit IgG FITC (Cat\#406403, Biolegend). Next, the cells were washed twice with FACS buffer, and 100,000-300,000 events were acquired on BD FACS celesta (BD Biosciences). Analysis was performed using the FlowJo software.

\section{RNA sequencing analysis}

Briefly, $5 \times 10^{6}$ CAR7 T cells/well were stimulated with Jurkat-GFP cells at a 4:1 ratio. The T cells were collected after $16 \mathrm{~h}$, and total RNA was isolated from each sample. Libraries were prepared using SMART-Seq v4 Ultra Low Input RNA Kit (Takara). PCR products were then indexed using Nextera XT DNA Library Prep Kit (Illumina) and sequenced across 75 base pairs (bp) using a paired-end strategy with a 150-cycle high-output flow cell on NextSeq 550 (Illumina). Fastq files from replicate sequencing runs were concatenated and aligned to hg38 using spliced transcript alignment to a reference (STAR) software version 2.5.2a. A mapped read depth of 60 million reads per sample was used. The reads were aligned to hg38 using the STAR alignment software.

\section{Detection of RD13-01 chromosomal translocation}

qPCR assays were used to detect the potential occurrence of a chromosomal translocation for RFX5, CD7, and TRAC. These six translocations were labeled as TRAC:CD7, TRAC:RFX5, CD7:TRAC, CD7:RFX5, RFX5:CD7, and RFX5:TRAC. The template contained tandem sequences for the four forward and four reverse primer-flanking sequences corresponding to the four probes. The concentration of the standard plasmid was determined using its stock solution, and its copy number was calculated accordingly. Genomic DNA was extracted using Fast Pure Cell/Tissue DNA Isolation Mini Kit (Vazyme Biotech). qPCR was performed, and the gene copy numbers of the genomic DNA were calculated according to the standard curve and $\mathrm{Cq}$ value.

\section{Trial design and oversight}

We conducted a first-in-human, single-arm, dose-escalation phase I clinical trial of a CD7 targeting "off-the-shelf" CAR-T cell (RD13-01) therapy for patients with $\mathrm{r} / / \mathrm{r}$ CD7 ${ }^{+}$hematological malignancies at the Bone Marrow Transplantation Center, the First Affiliated Hospital, Zhejiang University School of Medicine. The primary objective of the study was to assess the safety of RD13-01, while the secondary objective was to assess its efficacy.

The study protocol was approved by the First Affiliated Hospital, School of Medicine, Zhejiang University Institutional Review Board and registered in ClinicalTrials.gov (NCT04538599). The 13 participants or their guardians provided written informed consent in accordance with the Declaration of Helsinki. Patient 11 (diagnosed with AML) also consented for compassionate use. One patient was excluded because of CNS involvement. A total of 12 patients were enrolled in this study. The enrolled patients received one infusion of RD13-01 between September 2020 and January 2021, except for the first enrolled patient who received split-dosing infusion for safety concerns. 
Patients were given a lymphodepleting condition regimen consisting of fludarabine, cyclophosphamide, and etoposide before the RD13-01 infusion. Patient number two received a second dose on day 22 due to suboptimal expansion and unsatisfactory clinical response.

\section{Assessment of toxic effects and response}

CRS and ICANS were graded according to the ASTCT consensus ${ }^{47}$. Other toxicities during and after therapy were assessed according to the National Institutes of Health Common Terminology Criteria for Adverse Events Version 5.0 (http://ctep.cancer.gov/). Therapy responses were assessed using flow cytometry as well as morphological and imaging analyses according to National Comprehensive Cancer Network Guidelines (http://www.nccn.org).

\section{RD13-01 manufacturing and expansion assessment}

Following RD13-01 infusion, serial peripheral blood samples were collected in K2EDTA BD vacutainer tubes (BD Biosciences). The persistence of RD13-01 in fresh peripheral blood was determined using flow cytometry and qPCR. Circulating RD13-01 expansion was measured based on the proportion of CAR ${ }^{+}$cells among $\mathrm{CD}^{-} 5^{+} \mathrm{CD}^{-} \mathrm{T}^{-}$ lymphocytes. CAR DNA copies were also evaluated as another method of determining RD13-01 expansion and persistence. Genomic DNA was extracted using AxyPrep Blood Genomic DNA Miniprep Kit (Axygen) from cryopreserved peripheral blood and bone marrow samples. qPCR was performed in triplicate using AceQ qPCR Probe Master Mix (Vazyme Biotech) on a CFX Connect ${ }^{\mathrm{TM}}$ real-time PCR system (Bio-Rad). Copy numbers per microgram of genomic DNA were calculated from a standard curve of 10 -fold serial dilutions of purified CAR plasmid containing 2 $\times 10^{1}-2 \times 10^{6}$ copies $/ \mu \mathrm{L}$.

\section{Statistical analysis}

The data cutoff date was March 31, 2021. Variables were tabulated and summarized with descriptive statistics. For the time-to-event analyses, the Kaplan-Meier method was used to describe the LFS. Serum concentrations of cytokines in clinical samples were compared using the Mann-Whitney method whereas other comparisons were performed using the Student's t-test. All P-values presented are two-tailed. P-values lower than 0.05 were considered statistically significant. Statistical analysis was performed using GraphPad Prism 7 software.

\section{Declarations}

\section{Acknowledgements}

This study was supported by the National Natural Science Foundation of China (grant No. 81730008, 81770201, 81870153), Key Project of Science and Technology Department of Zhejiang Province (grant No. 2019C03016, 2018C03016-2, 2021C03010).

\section{Author Contributions}

Y.H., Y.Z., J.R. and H.H. designed the overall study and wrote the manuscript. Y.H., M.Z., H.Z., G.W., and H.H. designed the clinical trial. J.R., Y.J., G.C., T.G., J.Z., H.Y., L.L., W.Z.,J.L.,M.G.,K.W.,W.G. and L.H. performed the experiments. ., G.M and Y.Z. were responsible for manufacturing and quality control of CAR T cells. Y.H., M.Z., G.W., H.Z. and W.W. performed the clinical trial. J.Z., Y.H. ,W.G.and H.Z. analyzed the data. H.H. and J.R. discussed the results and manuscript. J.R. and H.H. supervised the study. All authors approved the article for submission and publication.

\section{Competing interests}

Page $13 / 25$ 


\section{References}

1. Litzow, M.R. \& Ferrando, A.A. How I treat T-cell acute lymphoblastic leukemia in adults. Blood 126, $833-841$ (2015).

2. Moskowitz, A.J., Lunning, M.A. \& Horwitz, S.M. How I treat the peripheral T-cell lymphomas. Blood 123, 26362644 (2014).

3. Cooper, M.L. \& DiPersio, J.F. Chimeric antigen receptor T cells (CAR-T) for the treatment of T-cell malignancies. Best Pract Res Clin Haematol 32, 101097 (2019).

4. Sánchez-Martínez, D., et al. Fratricide-resistant CD1a-specific CAR T cells for the treatment of cortical T-cell acute lymphoblastic leukemia. Blood 133, 2291-2304 (2019).

5. Maude, S.L., et al. Tisagenlecleucel in Children and Young Adults with B-Cell Lymphoblastic Leukemia. N Engl J Med 378, 439-448 (2018).

6. Schuster, S.J., et al. Tisagenlecleucel in Adult Relapsed or Refractory Diffuse Large B-Cell Lymphoma. N Engl J Med 380, 45-56 (2019).

7. Neelapu, S.S., et al. Axicabtagene Ciloleucel CAR T-Cell Therapy in Refractory Large B-Cell Lymphoma. N Engl J Med 377, 2531-2544 (2017).

8. Wang, M., et al. KTE-X19 CAR T-Cell Therapy in Relapsed or Refractory Mantle-Cell Lymphoma. N Engl J Med $382,1331-1342$ (2020).

9. Abramson, J.S., et al. Lisocabtagene maraleucel for patients with relapsed or refractory large B-cell lymphomas (TRANSCEND NHL 001): a multicentre seamless design study. The Lancet 396, 839-852 (2020).

10. Gomes-Silva, D., et al. CD7-edited T cells expressing a CD7-specific CAR for the therapy of T-cell malignancies. Blood 130, 285-296 (2017).

11. Fleischer, L.C., Spencer, H.T. \& Raikar, S.S. Targeting T cell malignancies using CAR-based immunotherapy: challenges and potential solutions. J Hematol Oncol 12, 141 (2019).

12. Depil, S., Duchateau, P., Grupp, S.A., Mufti, G. \& Poirot, L. 'Off-the-shelf' allogeneic CAR T cells: development and challenges. Nat Rev Drug Discov 19, 185-199 (2020).

13. Benjamin, R., et al. Genome-edited, donor-derived allogeneic anti-CD19 chimeric antigen receptor T cells in paediatric and adult B-cell acute lymphoblastic leukaemia: results of two phase 1 studies. Lancet 396, 18851894 (2020).

14. Hu, Y., et al. CRISPR/Cas9-Engineered Universal CD19/CD22 Dual-Targeted CAR-T Cell Therapy for Relapsed/Refractory B-cell Acute Lymphoblastic Leukemia. Clin Cancer Res (2021).

15. Li, S., et al. Eradication of T-ALL Cells by CD7-targeted Universal CAR-T Cells and Initial Test of Ruxolitinib-based CRS Management. Clin Cancer Res (2020).

16. Rosshart, S., et al. Interaction of KLRG1 with E-cadherin: new functional and structural insights. Eur J Immunol 38, 3354-3364 (2008).

17. Kaartinen, T., et al. Low interleukin-2 concentration favors generation of early memory T cells over effector phenotypes during chimeric antigen receptor T-cell expansion. Cytotherapy 19, 1130 (2017).

18. Zhang, X., Lv, X. \& Song, Y. Short-term culture with IL-2 is beneficial for potent memory chimeric antigen receptor T cell production. Biochem Biophys Res Commun 495, 1833-1838 (2018). 
19. Manz, M.G. Human-hemato-lymphoid-system mice: opportunities and challenges. Immunity 26, 537-541 (2007).

20. Saxena, A., et al. Biologic and clinical significance of CD7 expression in acute myeloid leukemia. Am J Hematol 58, 278-284 (1998).

21. Chang, H., Yeung, J., Brandwein, J. \& Yi, Q.L. CD7 expression predicts poor disease free survival and postremission survival in patients with acute myeloid leukemia and normal karyotype. Leukemia research 31, 157162 (2007).

22. Venditti, A., et al. Prognostic relevance of the expression of Tdt and CD7 in 335 cases of acute myeloid leukemia. Leukemia 12, 1056-1063 (1998).

23. Gomes-Silva, D., et al. CD7 CAR T Cells for the Therapy of Acute Myeloid Leukemia. Mol Ther 27, 272-280 (2019).

24. Fraietta, J.A., et al. Determinants of response and resistance to CD19 chimeric antigen receptor (CAR) T cell therapy of chronic lymphocytic leukemia. Nat Med 24, 563-571 (2018).

25. Ebi, H., et al. PI3K regulates MEK/ERK signaling in breast cancer via the Rac-GEF, P-Rex1. Proceedings of the National Academy of Sciences 110, $21124-21129$ (2013).

26. Verma, V., et al. MEK inhibition reprograms CD8+ T lymphocytes into memory stem cells with potent antitumor effects. Nature Immunology 22, 53-66 (2021).

27. Gornalusse, G.G., et al. HLA-E-expressing pluripotent stem cells escape allogeneic responses and lysis by NK cells. Nature biotechnology 35, 765 (2017).

28. Rettman, P., et al. New insights on the natural killer cell repertoire from a thorough analysis of cord blood cells. $J$ Leukoc Biol 100, 471-479 (2016).

29. Ma, M., et al. NKG2C+ NKG2A- natural killer cells are associated with a lower viral set point and may predict disease progression in individuals with primary HIV infection. Frontiers in immunology 8, 1176 (2017).

30. Xu, B., et al. An oncolytic herpesvirus expressing E-cadherin improves survival in mouse models of glioblastoma. Nature biotechnology 37, 45-54 (2019).

31. Wu, W., et al. Multiple Signaling Roles of CD3ع and Its Application in CAR-T Cell Therapy. Cell 182, $855-871$. e823 (2020).

32. Fajgenbaum, D.C. \& June, C.H.J.N.E.J.o.M. Cytokine storm. New England Journal of Medicine 383, 2255-2273 (2020).

33. Giavridis, T., et al. CAR T cell-induced cytokine release syndrome is mediated by macrophages and abated by IL1 blockade. Nat Med 24, 731-738 (2018).

34. Sachdeva, M., Duchateau, P., Depil, S., Poirot, L. \& Valton, J. Granulocyte-macrophage colony-stimulating factor inactivation in CAR T-cells prevents monocyte-dependent release of key cytokine release syndrome mediators. $J$ Biol Chem 294, 5430-5437 (2019).

35. Wu, W., et al. TLR ligand induced IL-6 counter-regulates the anti-viral CD8(+) T cell response during an acute retrovirus infection. Sci Rep 5, 10501 (2015).

36. Liu, Y., et al. Gasdermin E-mediated target cell pyroptosis by CAR T cells triggers cytokine release syndrome. Science immunology 5(2020).

37. McNamara, M.J., Kasiewicz, M.J., Linch, S.N., Dubay, C. \& Redmond, W.L.J.J.f.i.o.c. Common gamma chain (үc) cytokines differentially potentiate TNFR family signaling in antigen-activated CD8+ T cells. Journal for immunotherapy of cancer 2, 1-15 (2014). 
38. Sutherland, A.P., et al. IL-21 promotes CD8+ CTL activity via the transcription factor T-bet. The Journal of Immunology 190, 3977-3984 (2013).

39. Hinrichs, C.S., et al. IL-2 and IL-21 confer opposing differentiation programs to CD8+ T cells for adoptive immunotherapy. Blood 111, 5326-5333 (2008).

40. Cifaldi, L., et al. Inhibition of natural killer cell cytotoxicity by interleukin-6: implications for the pathogenesis of macrophage activation syndrome. Arthritis \& rheumatology 67, 3037-3046 (2015).

41. Heslop, H.E. How I treat EBV lymphoproliferation. Blood 114, 4002-4008 (2009).

42. Styczynski, J., et al. Management of Epstein-Barr Virus infections and post-transplant lymphoproliferative disorders in patients after allogeneic hematopoietic stem cell transplantation: Sixth European Conference on Infections in Leukemia (ECIL-6) guidelines. Haematologica 101, 803-811 (2016).

43. San-Juan, R., et al. Epstein-Barr virus-related post-transplant lymphoproliferative disorder in solid organ transplant recipients. Clin Microbiol Infect 20 Suppl 7, 109-118 (2014).

44. Pina-Oviedo, S., Miranda, R.N. \& Medeiros, L.J. Cancer Therapy-associated Lymphoproliferative Disorders: An Under-recognized Type of Immunodeficiency-associated Lymphoproliferative Disorder. Am J Surg Patho/ 42, 116-129 (2018).

45. O'Mahony, D., et al. EBV-Related Lymphoproliferative Disease Complicating Therapy with the Anti-CD2 Monoclonal Antibody, Siplizumab, in Patients with T-Cell Malignancies. Clinical Cancer Research 15, $2514-2522$ (2009).

46. Zettl, A., et al. Epstein-Barr virus-associated B-cell lymphoproliferative disorders in angloimmunoblastic T-cell lymphoma and peripheral T-cell lymphoma, unspecified. Am J Clin Pathol 117, 368-379 (2002).

47. Greco, A., et al. Altered urinary excretion of human kininase activity in hypertension. Adv Exp Med Bio/ 120B, 645-649 (1979).

\section{Table 1}

Table 1 Patient baseline characteristics and clinical outcomes. 


\begin{tabular}{|c|c|c|c|c|c|c|c|c|c|c|c|}
\hline $\begin{array}{l}\text { Patient } \\
\text { No. }\end{array}$ & $\begin{array}{l}\text { Age } \\
\text { (year) }\end{array}$ & Sex & Disease & $\begin{array}{l}\text { Prior } \\
\text { SCT }\end{array}$ & $\begin{array}{l}\text { Prior } \\
\text { CNSL }\end{array}$ & EMD & $\begin{array}{l}\text { Blast } \\
\text { cell } \\
\text { in } \\
\text { BM }\end{array}$ & $\begin{array}{l}\text { CAR-T } \\
\text { dose } \\
\left(10^{7} / \mathrm{kg}\right)\end{array}$ & CRS & ICANS & $\begin{array}{l}\text { Clinical } \\
\text { out } \\
\text { come } \\
\text { at day } \\
28\end{array}$ \\
\hline 1 & 54 & $\mathrm{M}$ & ETP & No & No & No & $10 \%$ & 1 & 2 & None & $\mathrm{CR}$ \\
\hline 2 & 36 & $\mathrm{~F}$ & T- ALL & Auto- & Yes & Breast & $21 \%$ & 1 & 0 & None & NR \\
\hline 3 & 62 & $\mathrm{~F}$ & T-LBL & No & No & $\begin{array}{l}\text { Breast, } \\
\text { cervical, } \\
\text { axillary and } \\
\text { inguinal lymph } \\
\text { node }\end{array}$ & $48 \%$ & 1 & 2 & None & NA \\
\hline 4 & 66 & $\mathrm{~F}$ & ETP & No & Yes & No & $37 \%$ & 2 & 2 & None & CR \\
\hline 5 & 33 & M & ETP & No & Yes & No & $95 \%$ & 2 & 2 & None & CR \\
\hline 6 & 34 & M & $\begin{array}{l}\text { PTCL, } \\
\text { NOS }\end{array}$ & No & No & $\begin{array}{l}\text { Cervical, } \\
\text { clavicle, breast } \\
\text { internal breast } \\
\text { arterial, } \\
\text { mediastinum, } \\
\text { retroperitoneal, } \\
\text { iliac vessel, } \\
\text { pelvic ingiliac } \\
\text { fossa inguinal } \\
\text { lymph node }\end{array}$ & $39 \%$ & 2 & 1 & None & CR \\
\hline 7 & 34 & M & $\begin{array}{l}\text { ETP } \\
\text { ALL }\end{array}$ & $\begin{array}{l}\text { Allo- } \\
\text { SCT }\end{array}$ & Yes & $\begin{array}{l}\text { Gum, anterior } \\
\text { sacral soft } \\
\text { tissue, } \\
\text { intercostal } \\
\text { muscle, } \\
\text { infraspinatus } \\
\text { muscle }\end{array}$ & BMR & 3 & 2 & None & NR \\
\hline 8 & 55 & $\mathrm{~F}$ & ETP & No & No & & $9 \%$ & 3 & 2 & None & CR \\
\hline 9 & 30 & M & T-ALL & $\begin{array}{l}\text { Allo- } \\
\text { SCT }\end{array}$ & No & $\begin{array}{l}\text { Mediastinal } \\
\text { soft tissue and }\end{array}$ & BMR & 2 & 2 & None & CR \\
\hline 10 & 23 & $\mathrm{~F}$ & $\begin{array}{l}\text { EBV+ } \\
\text { NK/TCL }\end{array}$ & No & No & $\begin{array}{l}\text { Skin and } \\
\text { subcutaneous } \\
\text { nodules, } \\
\text { inguinal lymph } \\
\text { nodes, spleen, } \\
\text { liver }\end{array}$ & $7 \%$ & 2 & 0 & None & PR \\
\hline 11 & 16 & M & AML- & No & No & No & $51 \%$ & 2 & 1 & None & CR \\
\hline 12 & 8 & M & T-LBL & No & No & $\begin{array}{l}\text { Mediastinal } \\
\text { soft tissue, } \\
\text { mesenteric } \\
\text { lymph nodes }\end{array}$ & $9 \%$ & 3 & 2 & None & PR \\
\hline
\end{tabular}

\section{Extended Tables}

Extended data Table 1 Infusion products of RD13-01 


\begin{tabular}{|c|c|c|c|c|c|c|}
\hline Patient No. & CAR-T batch No. & Viability (\%) & CD4:CD8 ratio (\%) & $\mathrm{CAR}^{+}(\%)$ & HLA $^{-}(\%)$ & $\mathrm{CD}^{-} \mathrm{CD}^{-}(\%)$ \\
\hline 1 & 2020062001 & \multirow[t]{3}{*}{74} & \multirow[t]{3}{*}{58} & \multirow[t]{3}{*}{62} & \multirow[t]{3}{*}{93} & \multirow[t]{3}{*}{99} \\
\hline 2 & 2020062001 & & & & & \\
\hline 3 & 2020062001 & & & & & \\
\hline 4 & C20201001 & \multirow[t]{3}{*}{95} & \multirow[t]{3}{*}{26} & \multirow[t]{3}{*}{59} & \multirow[t]{3}{*}{94} & \multirow[t]{3}{*}{80} \\
\hline 5 & C20201001 & & & & & \\
\hline 6 & C20201001 & & & & & \\
\hline 7 & C20201101-01 & \multirow[t]{2}{*}{93} & \multirow[t]{2}{*}{39} & \multirow[t]{2}{*}{69} & \multirow[t]{2}{*}{96} & \multirow[t]{2}{*}{81} \\
\hline 8 & C20201101-01 & & & & & \\
\hline 9 & C20201101-02 & \multirow[t]{2}{*}{93} & \multirow[t]{2}{*}{57} & \multirow[t]{2}{*}{67} & \multirow[t]{2}{*}{95} & \multirow[t]{2}{*}{81} \\
\hline 10 & C20201101-02 & & & & & \\
\hline \multirow[t]{2}{*}{$11^{*}$} & 2020062001 & 74 & 58 & 62 & 93 & 99 \\
\hline & C20201001 & 95 & 26 & 59 & 94 & 80 \\
\hline 12 & C20201101-02 & 93 & 57 & 67 & 95 & 81 \\
\hline
\end{tabular}

*Patient 11 received 2020062001 RD13-01 cells and C20201001 RD13-01 cells in a ratio of 5.2:1.

Extended data Table 2. Prior lines of therapy for each patient

\begin{tabular}{|c|c|c|}
\hline $\begin{array}{l}\text { Patient } \\
\text { No. }\end{array}$ & $\begin{array}{l}\text { Prior lines of } \\
\text { therapy }\end{array}$ & Prior therapy \\
\hline 1 & 4 & $\begin{array}{lllllllllll}\begin{array}{l}\text { VDCLP, VP+Hyper } \\
\text { CHG+venectoclax }\end{array} & \text { CVAD } & \text { part } & \text { B, Hyper } & \text { CVAD } & \text { part } & \text { B } & \text { CAG } & \text { CAG } \\
\end{array}$ \\
\hline 2 & 5 & $\begin{array}{cccc}\text { Hyper-CVAD partA, CAG } & \text { ASCT, } & \text { CAG+venectoclax, } \\
\text { IDA+VDS+IFO+VP16+Methylprednisolone } & \end{array}$ \\
\hline 3 & 4 & $\begin{array}{l}\text { Hyper-CVAD part } \\
\text { CHG+venectoclax }\end{array}$ \\
\hline 4 & 4 & 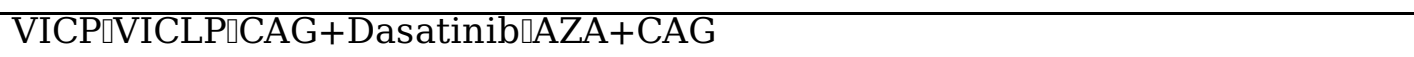 \\
\hline 5 & 5 & $\begin{array}{l}\text { VDCP, HyperCVAD part A, CAG, Hyper CVAD partB, VICP+Temozolomide, } \\
\text { intrathecal injection, VICD }\end{array}$ \\
\hline 6 & 4 & CHOPE $₫ A Z A+G M D \square A Z A+C h i d a m i d e+G M D \square V I D E R$ \\
\hline 7 & 7 & VDLP, CAG, CHAG, allo-HSCT $\mathrm{intrathecal} \mathrm{injection} \square$ radiotherapy $\square$ DLI \\
\hline 8 & 2 & VDCP, VDCLP \\
\hline 9 & 4 & CHOP, VDCLP, Hyper CVAD part A, HyperCVAD part B, allo-HSCT \\
\hline 10 & 2 & VP16+DXM, P-Gemox \\
\hline 11 & 2 & IA, HD-Arac \\
\hline 12 & 6 & 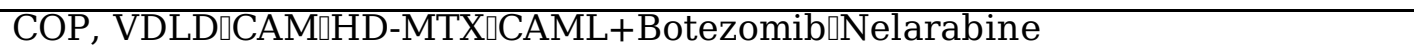 \\
\hline
\end{tabular}

Allo-HSCT: allogeneic hematopoietic stem cell transplantation; ASCT: autologous stem cell transplantation; AZA: 5-azacytidine; CAG: cytarabine, aclacinomycin, granulocyte-colony stimulating factor; CAM: cyclophosphamide, cytarabine, mercaptopurine; CAML: cyclophosphamide, cytarabine, mercaptopurine, l-asparaginase; CHAG: cytarabine, harringtonine, aclacinomycin, granulocyte-colony stimulating factor; CHG: cytarabine, harringtonine, granulocyte-colony stimulating factor; CHOP: vincristine, daunorubicin, cyclophosphamide, prednisone; CHOPE: vincristine, daunorubicin, cyclophosphamide, prednisone, etoposide; COP: vincristine, cyclophosphamide, prednisone; DLI: donor lymphocyte infusion; DXM: dexamethasone; GMD: gemcitabine, oxaliplatin, dexamethasone; HD-Arac: high dose cytarabine; HD-MTX: high dose methotrexate; Hyper CVAD part A: vincristine, cyclophosphamide, doxorubicin, dexamethasone; Hyper CVAD part B: methotrexate, cytarabine; IA: idarubicin, cytarabine; IDA: idarubicin; IFO: ifosphamide; P-Gemox: pegaspargase, gemcitabine, 
oxaliplatin; VDCLP: vincristine, daunorubicin, cyclophosphamide, l-asparaginase, prednisone; VDCP: vincristine, daunorubicin, cyclophosphamide, prednisone; VDLD: vincristine, daunorubicin, 1asparaginase, dexamethasone; VDLP: vincristine, daunorubicin, l-asparaginase, prednisone; VDS: vindesin; VICD: vincristine, idarubicin, cyclophosphamide, dexamethasone; VICLP: vincristine, idarubicin, cyclophosphamide, L-asparaginase, prednisone; VICP: vincristine, idarubicin, cyclophosphamide, prednisone; VIDER: vincristine, idarubicin, dexamethasone, etoposide, lenalidomide; VIP: vincristine, idarubicin, cyclophosphamide, prednisone; VP: vincristine, prednisone; VP16: etoposide.

Extended data Table 3. Summary of adverse events in the study 


\begin{tabular}{|c|c|c|c|}
\hline Adverse events & All grades & Grade1/2 & Grade3/4 \\
\hline Hypotension & 12 & 11 & 1 \\
\hline Anemia & 12 & 2 & 10 \\
\hline Neutrophil count decreased & 12 & 0 & 12 \\
\hline Platelet count decreased & 12 & 0 & 12 \\
\hline Fever & 11 & 11 & 0 \\
\hline Malaise & 11 & 11 & 0 \\
\hline Chills & 10 & 10 & 0 \\
\hline Cytokine release syndrome & 10 & 10 & 0 \\
\hline Cough & 8 & 8 & 0 \\
\hline Mucositis oral & 8 & 7 & 1 \\
\hline Nausea & 8 & 7 & 1 \\
\hline Aspartate aminotransferase increased & 8 & 6 & 2 \\
\hline Hypocalcemia & 7 & 7 & 0 \\
\hline Alanine aminotransferase increased & 7 & 4 & 3 \\
\hline Abdominal pain & 6 & 6 & 0 \\
\hline Headache & 6 & 6 & 0 \\
\hline Purpura & 6 & 5 & 1 \\
\hline Hypokalemia & 6 & 3 & 3 \\
\hline Anorexia & 5 & 5 & 0 \\
\hline Bloating & 5 & 5 & 0 \\
\hline Constipation & 5 & 5 & 0 \\
\hline Diarrhea & 5 & 5 & 0 \\
\hline Vomiting & 5 & 5 & 0 \\
\hline Hyperglycemia & 5 & 3 & 2 \\
\hline Gum infection & 5 & 2 & 3 \\
\hline Dizziness & 4 & 4 & 0 \\
\hline Hyponatremia & 4 & 4 & 0 \\
\hline Edema limbs & 3 & 3 & 0 \\
\hline Hypophosphatemia & 3 & 3 & 0 \\
\hline Insomnia & 3 & 3 & 0 \\
\hline Pleural effusion & 3 & 3 & 0 \\
\hline Productive cough & 3 & 3 & 0 \\
\hline Pruritus & 3 & 3 & 0 \\
\hline Rash maculo-papular & 3 & 3 & 0 \\
\hline Hypoxia & 3 & 1 & 2 \\
\hline Anal mucositis & 2 & 2 & 0 \\
\hline Blood bilirubin increased & 2 & 2 & 0 \\
\hline Dysphagia & 2 & 2 & 0 \\
\hline Edema face & 2 & 2 & 0 \\
\hline Epistaxis & 2 & 2 & 0 \\
\hline Hemorrhoids & 2 & 2 & 0 \\
\hline Hyperhidrosis & 2 & 2 & 0 \\
\hline Hyperphosphatemia & 2 & 2 & 0 \\
\hline Hyperuricemia & 2 & 2 & 0 \\
\hline Palpitation & 2 & 2 & 0 \\
\hline Pharyngolaryngeal pain & 2 & 2 & 0 \\
\hline Pneumonitis & 2 & 0 & 2 \\
\hline Sepsis & 2 & 0 & 2 \\
\hline Anal pain & 1 & 1 & 0 \\
\hline Anal fissure & 1 & 1 & 0 \\
\hline Ascites & 1 & 1 & 0 \\
\hline Chest wall pain & 1 & 1 & 0 \\
\hline Depression & 1 & 1 & 0 \\
\hline Facial pain & 1 & 1 & 0 \\
\hline Gallbladder pain & 1 & 1 & 0 \\
\hline Gingival pain & 1 & 1 & 0 \\
\hline Hemorrhoidal hemorrhage & 1 & 1 & 0 \\
\hline Hypertriglyceridemia & 1 & 1 & 0 \\
\hline
\end{tabular}

Page 20/25 


\begin{tabular}{cccc} 
Hypoglycemia & 1 & 1 & 0 \\
\hline Neck edema & 1 & 1 & 0 \\
\hline Non-cardiac chest pain & 1 & 1 & 0 \\
\hline Oral hemorrhage & 1 & 1 & 0 \\
\hline Pain of skin & 1 & 1 & 0 \\
\hline Pericardial effusion & 1 & 1 & 0 \\
\hline Periorbital edema & 1 & 1 & 0 \\
\hline Urinary frequency & 1 & 1 & 0 \\
\hline Urinary tract pain & 1 & 1 & 0 \\
\hline Dyspnea & 1 & 0 & 1 \\
\hline Hypernatremia & 1 & 0 & 1 \\
\hline Laryngeal edema & 1 & 0 & 1 \\
\hline Laryngitis & 1 & 0 & 1 \\
\hline Pharyngitis & 1 & 0 & 1 \\
\hline Respiratory failure & 1 & 0 & 1 \\
\hline Second primary malignancy & 1 & 0 & 1 \\
\hline Total & 279 & 215 & 64 \\
\hline
\end{tabular}

\section{Figures}

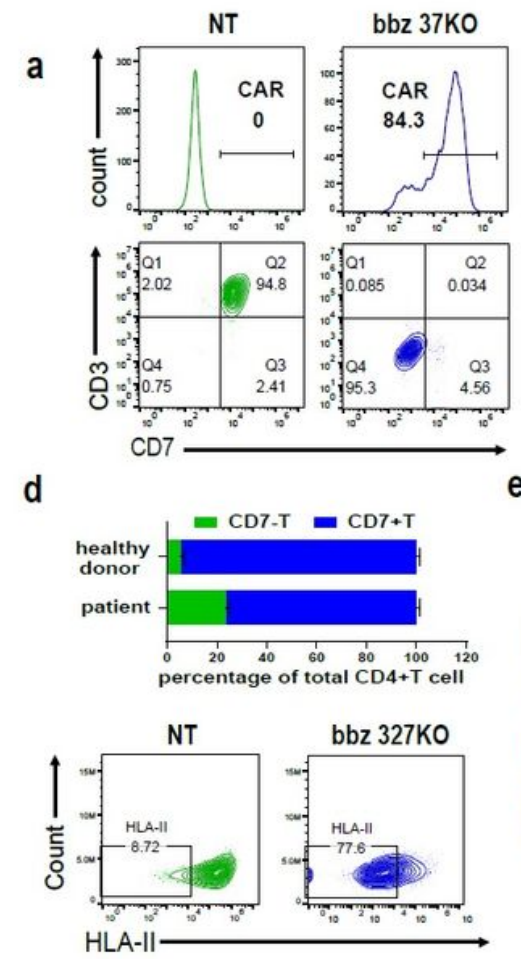

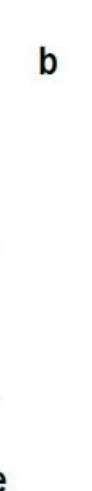

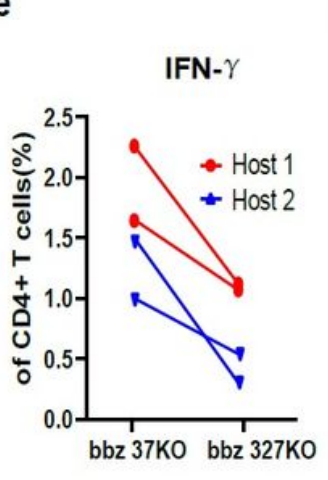

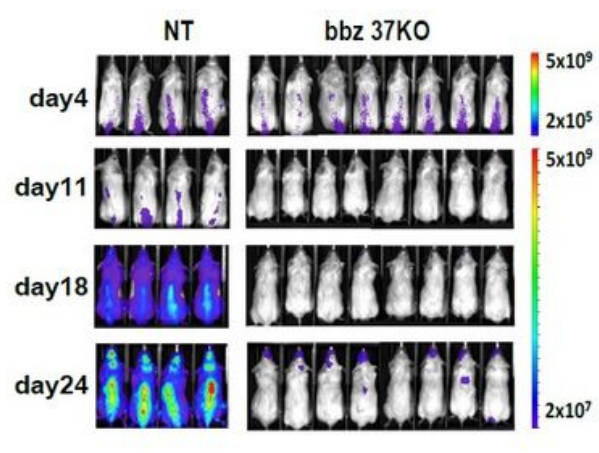

f

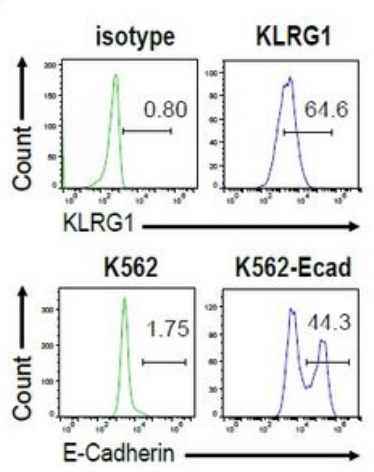

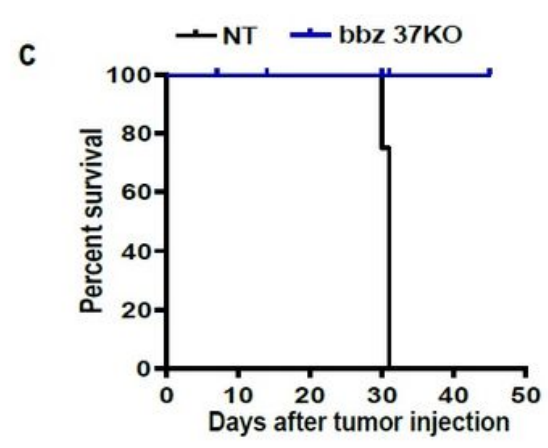

g

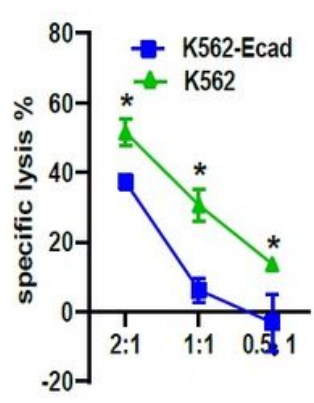

IFN- $\gamma$

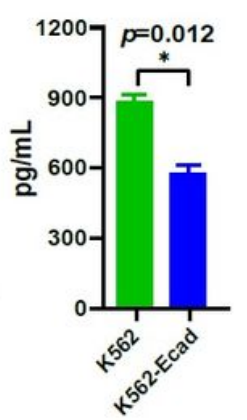

Figure 1

Construction of rejection-resistant "off-the-shelf" anti-CD7 CAR-T cells. a. TCR- and CD7-ablated anti-CD7 CAR (bbz 37KO CAR7) T cells were generated using CRISPR/Cas9 gene editing. CAR expression and gene disruption efficiency of TCR/CD3 and CD7 was measured using flow cytometry. b. Tumor control ability of CAR7 T cells was evaluated in a Jurkat tumor mouse model. NCG mice were injected with $1 \times 106$ Jurkat tumor cells intravenously (i.v.) and 
randomly divided into two groups based on the luciferase intensity measurements performed on day 3 . The mice were treated with $10 \times 106$ CAR7 T cells (single injection). Mice treated with non-transduced T (NT) cells from the same donor served as control. Images were obtained from the surviving animals as indicated. Imaging commenced one day before the start of T cell treatment $(N T, n=4 ; b b z 37 K O, n=8)$. c. Survival curve of mice bearing Jurkat tumor treated with either NT or CAR7 T cells $(N T, n=4 ; b b z 37 K O, n=8)$. $d$. CD7 expression on CD4 T cells was determined in healthy individuals and patient samples. HLA-II expression was eliminated by disrupting RFX5 in $37 \mathrm{KO}$ CAR7 T cells. e. INF-y production by allogeneic CD4 T cells toward 37KO and 327 KO CAR7 T cells were detected using flow cytometry $(n=2)$. f. KLRG1 expression on NK cells and engineered expression of E-cadherin on K562 (K562-ECad) cells. g. NK cell cytotoxicity toward K562 and K562-ECad cells was detected using a luciferase-based killing assay $(n=3)$ and by measuring INF-y production $(n=3)$. All comparisons were determined using t-test. *, $P<$ $0.05 ; * \star, P<0.01 ; n s$, not significant.

a

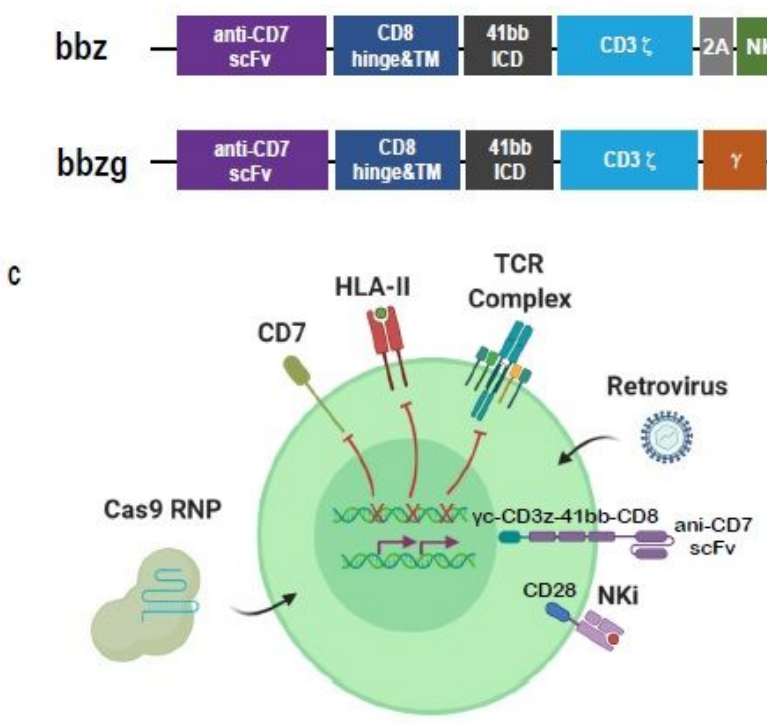

g
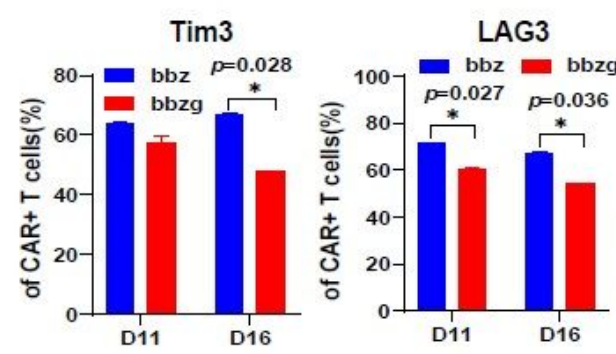

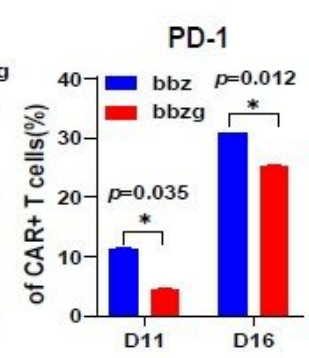

b
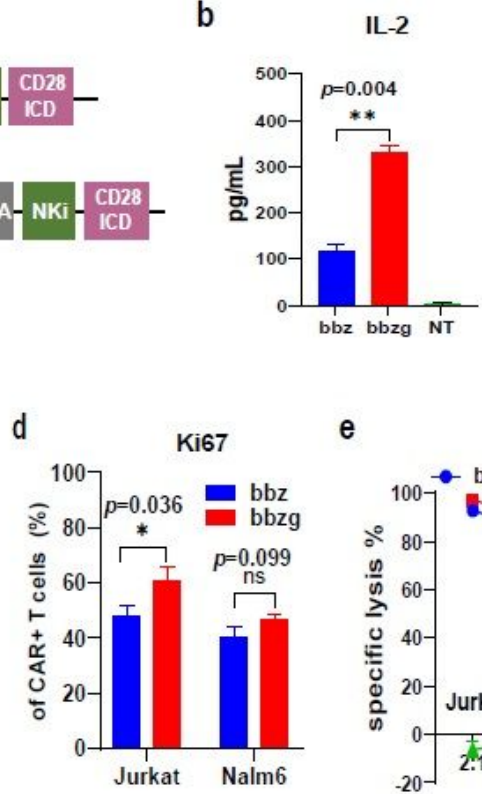

e
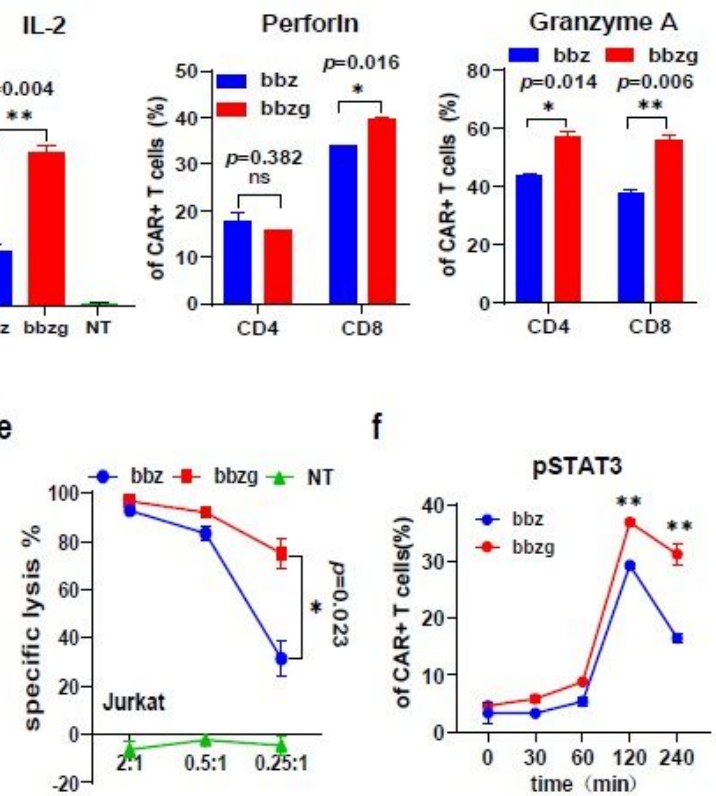

h

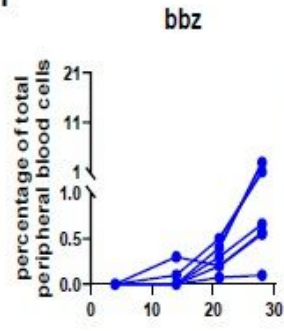

bbzg

NT

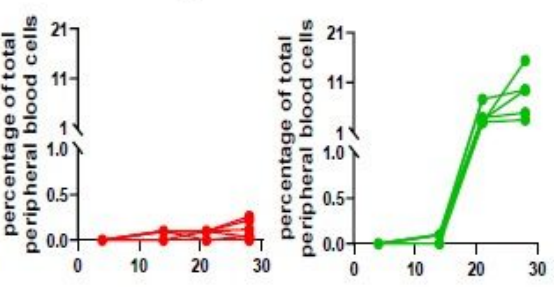

\section{Figure 2}

Addition of yc improves CAR-T cell function. a. Schematic illustration of yc-tethered bbzg-CAR design. b. Cytokine secretion and effector formation augmentation in bbzg-CAR-T cells following Jurkat tumor cell challenge, compare with bbz-CAR-T cells $(n=3)$. c. Illustration of rejection-resistant CAR7bbzg-NKi T cells. d. Ki67 staining of bbz and bbzg-CAR-T cells $(n=3)$. Cytolytic function and phenotype of bbzg-CAR-T cells were evaluated based on e.

cytotoxicity; f. phosphorylation of STAT3 after antigen stimulation, and g. expression of exhaustion markers Tim3, LAG3, and PD-1 ( $n=3$ ). Tumor control ability of bbzg-CAR-T cells was evaluated in a Jurkat tumor mouse model. NCG mice were injected with $1 \times 106$ Jurkat tumor cells (i.v.) and randomly divided into three groups based on the 
luciferase intensity measured on day 3 . Mice were treated with $10 \times 106$ CAR7 (single injection). Mice treated with NT cells from the same donor served as control. h. Residual tumor in $327 \mathrm{KO}$ bbz- and bbzg-CAR-T cell-treated Jurkat tumor-bearing mice were measured using flow cytometry (NT, $n=5 ; b b z, n=6 ;$ bbzg, $n=6)$. All comparisons were determined using t-test. *, $\mathrm{P}<0.05 ;$ **, $\mathrm{P}<0.01$; ns, not significant.

a

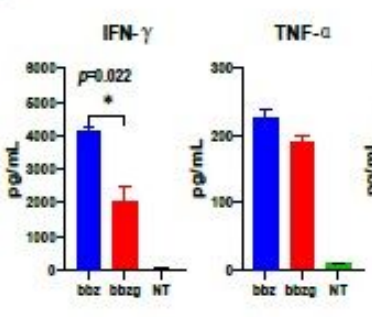

C
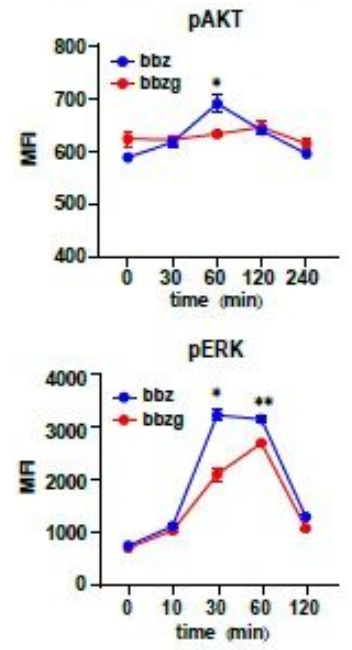

e
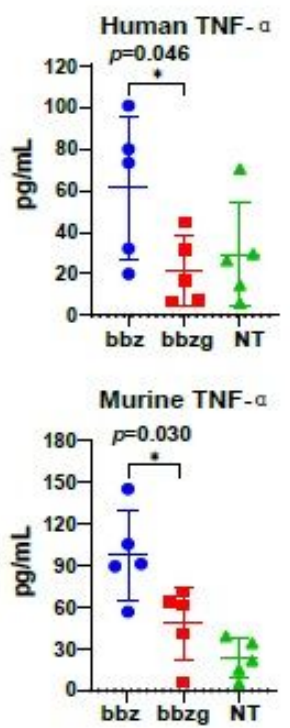
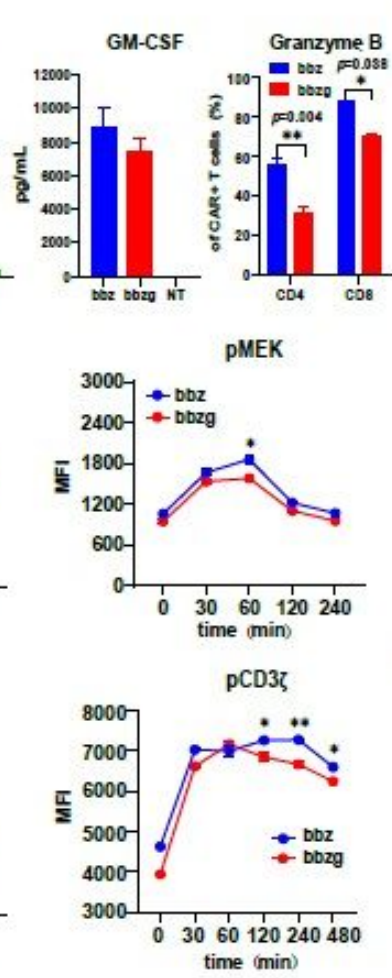

b

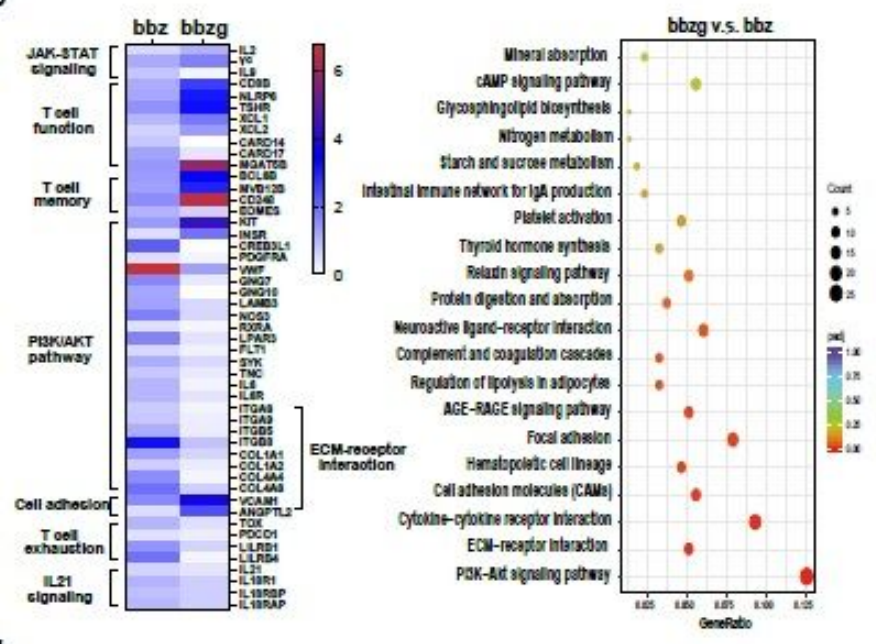

d
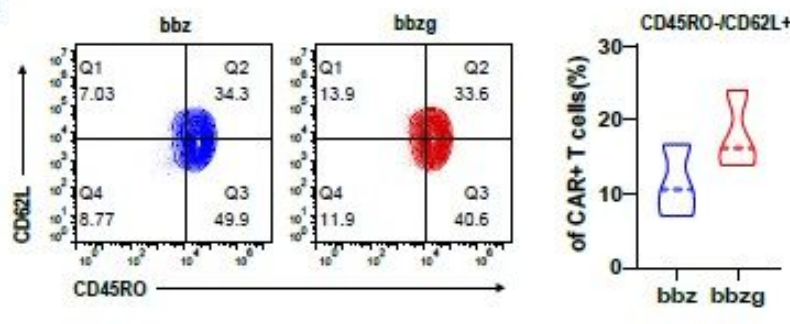
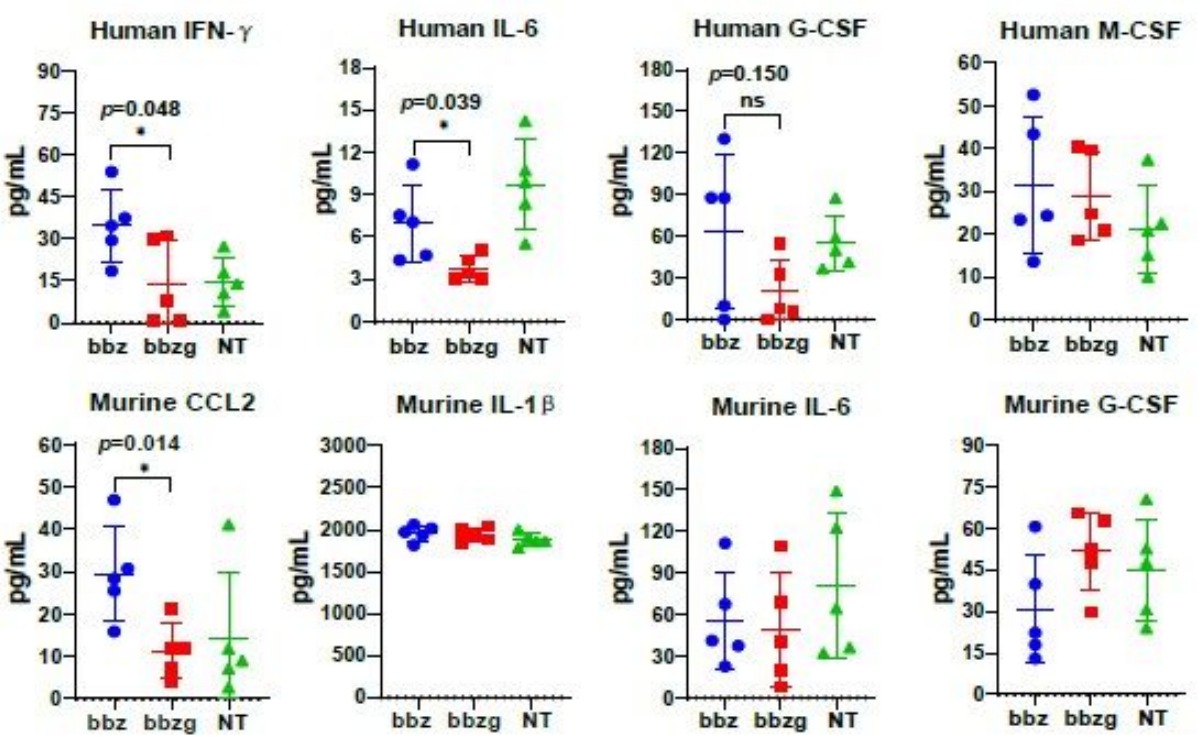

\section{Figure 3}

Addition of yc decreases CRS-related cytokine production by suppressing the PI3K/AKT pathway. a. Release of IFN- $\gamma$, TNF-a, GM-CSF, and granzyme B by $327 \mathrm{KO}$ bbz- and bbzg-CAR-T cells following Jurkat cell stimulation $(n=3)$. $b$. Differential gene expression and pathway analysis in $327 \mathrm{KO}$ bbz- and bbzg-CAR-T cells after Jurkat cell stimulation. c. phosphorylation of AKT, MEK, ERK and CD3Z after antigen stimulation ( $n=3)$. d. Phenotype of $327 K O$ bbz- and bbzg-CAR-T cells. CD62L+/CD45RO- stem cell memory cell population $(n=3)$. e. Cytokine release profile of $327 \mathrm{KO}$ 
bbz- and bbzg-CAR-T cells in a humanized Jurkat tumor-bearing NCG mouse model. Levels of human TNF- $a$, IFN- $\gamma$, IL-6, G-CSF, and M-CSF were measured using ELISA. Murine TNF-a, CCL2, IL-1 $\beta$, IL-6, and G-CSF levels were also measured $(n=5)$. All comparisons were determined using t-test. * $P<0.05$; ${ }^{*}, P<0.01$; ns, not significant.
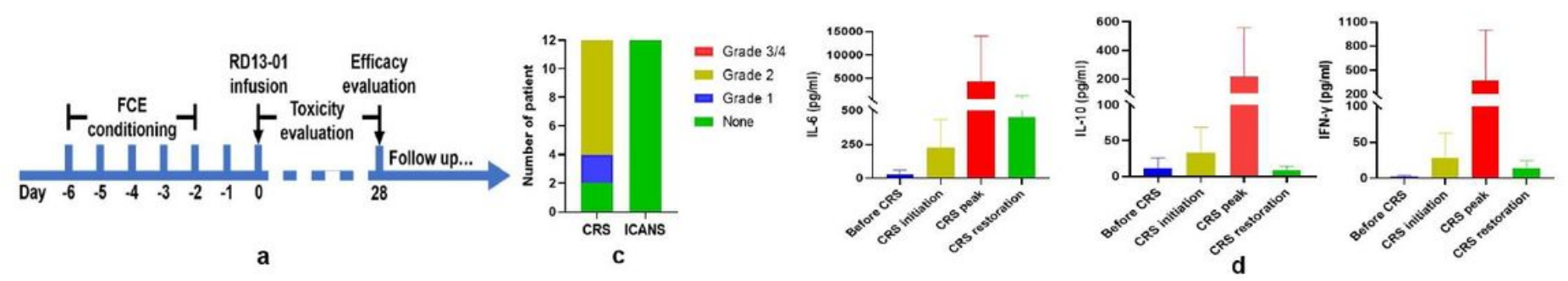

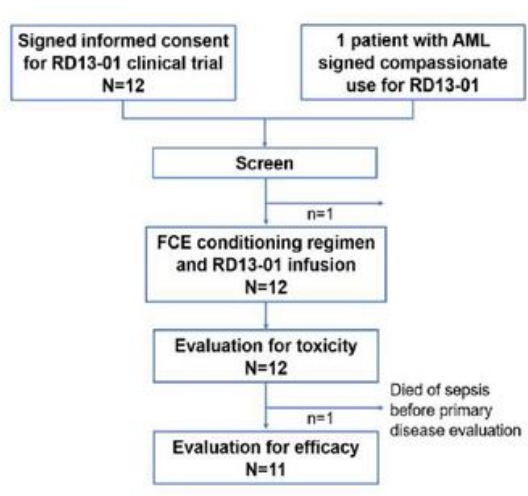

b

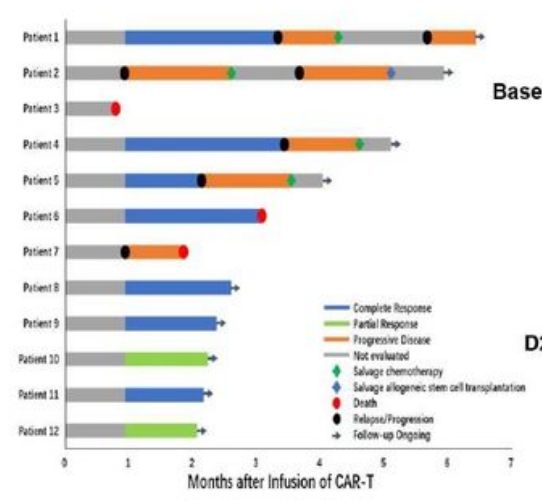

e

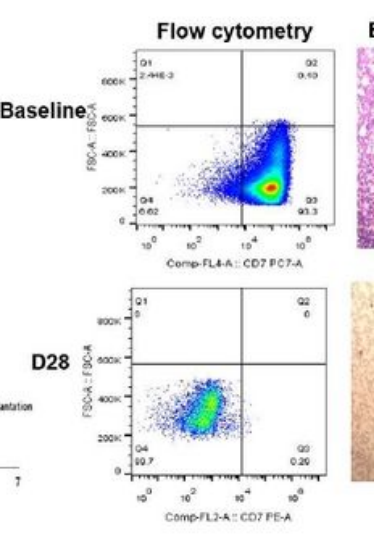

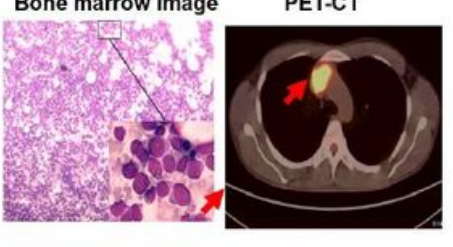

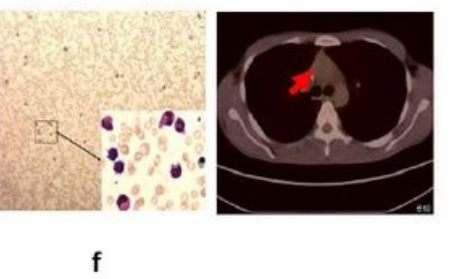

\section{Figure 4}

Clinical procedure and outcomes of treatment with RD13-01 CAR-T cells. a. Clinical treatment protocol: the day of RD13-01 infusion was set as day 0; the lymphodepleting condition regimen consisted of fludarabine $30 \mathrm{mg} / \mathrm{m} 2 \mathrm{on}$ day -7 to -3 , cyclophosphamide $300 \mathrm{mg} / \mathrm{m} 2$ on day -7 to -3 , and etoposide $100 \mathrm{mg} /$ on day -7 to -3 . b. Patient enrollment flow chart. c. Incidence of CRS and ICANS. d. Serum concentrations of CRS-related cytokines IL-6, IL-10, and IFN- $\gamma$ in patients who developed CRS after RD13-01 infusion. IL-6, IL-10, and IFN- $\gamma$ levels compared with those before CRS developed. ${ }^{*} P<0.05$; ${ }^{*} P<0.01$. e. Swimmer plot $(n=12)$, in which each bar represents an individual patient. Responses on day 28 are indicated by different colors (blue, CR; green, PR; orange, PD; gray, NE). Bars with solid arrows represent patients in an ongoing follow-up. f. Flow cytometry, bone marrow imaging, and positron emission tomography-computed tomography were used to evaluate the responses following RD13-01 infusion; left and middle, CD7+ malignant cell count in bone marrow measured using flow cytometry and microscopy decreased dramatically after RD13-01 infusion in patient 5; computed tomography scans show complete response to RD13-01 in patient 9; leukemic cells and lymphoma are indicated by red arrows. 

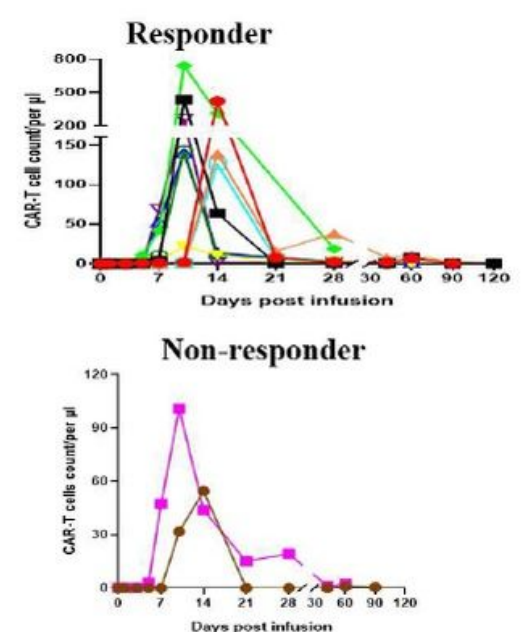

a

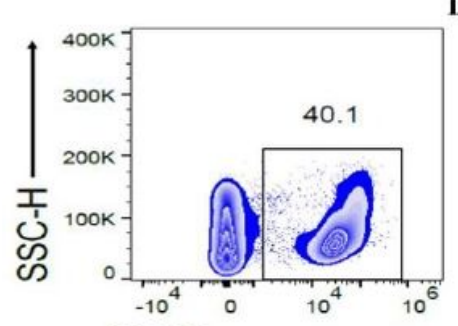

CD45

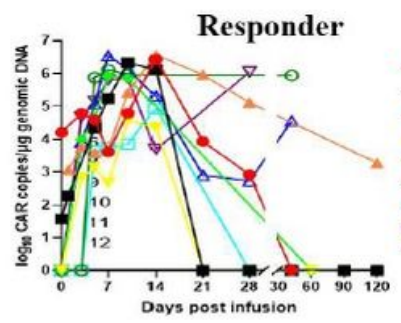

Non-responder

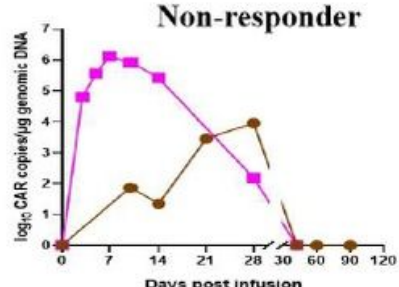

b

Day 15

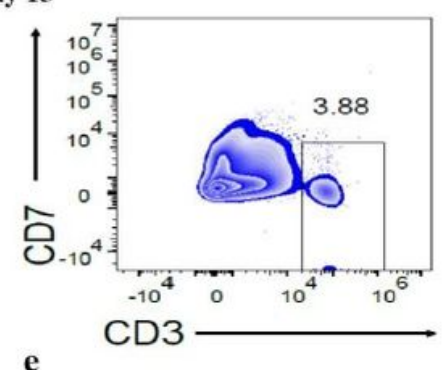

Patient 1 Patient 2 Patient 3 Patient 4 Patient 5 Patient 6

Patient 8

Patient 9

Patient 10

Patient 11

Patient 12

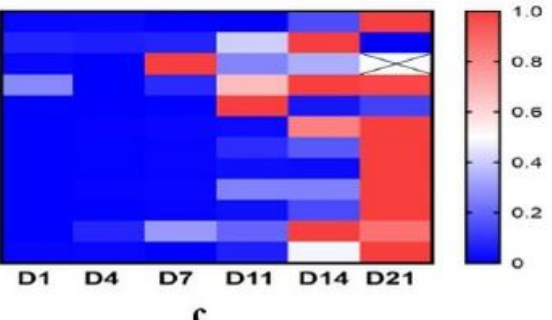

c

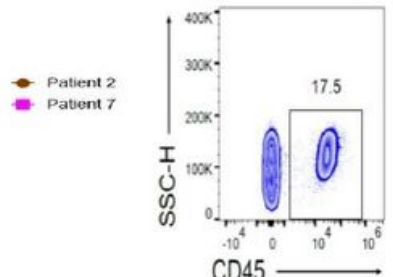

CD45
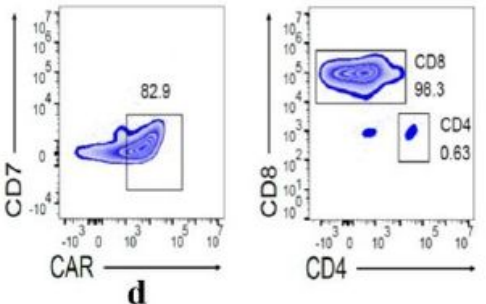

Day 62
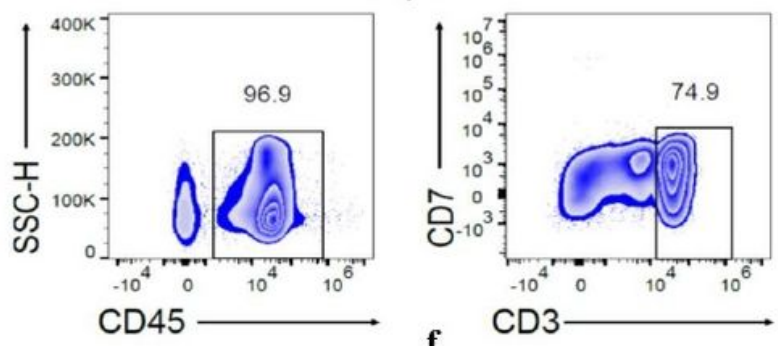

Figure 5

RD13-01 expansion in vivo and CD7- T cell compensation. a. Absolute count of RD13-01 cells in peripheral blood measured using flow cytometry divided by the 28-day response. Expansion of RD13-01 was not observed in patient 2, who did not survive 28 days; thus, the response was not evaluated. b. Expansion of RD13-01 in peripheral blood measured using qPCR divided by the 28-day response. The horizontal line at 20 copies/ $\mu \mathrm{g}$ genomic DNA represents the lower limit of quantification in the assay. c. Heatmap showing that intrinsic CD7- T cell count in peripheral blood increased on days 1, 4, 7, 11, 14, and 28 after RD13-01 inoculation. Color scheme is based on the normalization of absolute count of CD7- T cells using the min-max method from 0 (white) to 1 (blue). X indicates loss of the sample on day 28 owing to death of patient 2 on day 24. d. CAR-T cell expansion measured using flow cytometry on day 15 in patient 5. e. Intrinsic CD7- T cell count started to increase on day 15 in patient 5. f. Intrinsic CD3+CD7- T cells were predominant among CD3+T cells on day 62 in patient 5 .

\section{Supplementary Files}

This is a list of supplementary files associated with this preprint. Click to download.

- ExtendedDataFig.1.pdf

- ExtendedDataFig.2.pdf

- ExtendedDataFig.3.pdf

- ExtendedFig.4.pdf 